\title{
Modelling a targeted use of pesticide procedure for pest populations with heterogeneous spatial distributions
}

\author{
by Petrovskaya, N.B., Ellis, J.R., Forbes, E. and Walters, \\ K.F.A.
}

Copyright, publisher and additional Information: This is the author accepted manuscript. The final published version (version of record) is available online via Elsevier.

This version is made available under the CC-BY-ND-NC licence:

https://creativecommons.org/licenses/by-nc-nd/4.0/legalcode

Please refer to any applicable terms of use of the publisher

DOI: https://doi.org/10.1016/j.ecolmodel.2020.109059

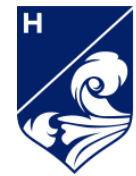

Harper Adams University

Petrovskaya, N.B., Ellis, J.R., Forbes, E. and Walters, K.F.A. 2020. Modelling a targeted use of pesticide procedure for pest populations with heterogeneous spatial distributions. Ecological Modelling. 
MODELLING A TARGETED USE OF PESTICIDE PROCEDURE FOR PEST POPULATIONS

WITH HETEROGENEOUS SPATIAL DISTRIBUTIONS

\author{
Natalia B. Petrovskaya ${ }^{a 1}$, John R. Ellis ${ }^{a}$, Emily Forbes ${ }^{b}$, Keith F.A. Walters ${ }^{b}$ \\ ${ }^{a}$ School of Mathematics, University of Birmingham, \\ Birmingham, B15 2TT, U.K. \\ ${ }^{b}$ Centre for Integrated Pest Management, Harper Adams University, Newport, Shropshire, \\ TF10 8NB U.K.
}

\begin{abstract}
Commercial and environmental considerations have led to increased pressure to reduce pesticide use in agricultural crops resulting in a growing interest in development of pesticide application protocols that allow for their targeted use. In this paper, we revisit a standard decision-making protocol for pesticide application and introduce protocol modifications to apply pesticide to selected spatial sub-domains in the agricultural field. The baseline case we consider is the control of populations of the grey field slug (Deroceras reticulatum) in commercialfields. It is well known that slugs have strongly heterogeneous (patchy) spatial distribution and we show that targeting patches with higher slug density only, may offer significant potential for reducing the use of pesticides. An approach to incorporating targeted application of pesticide into a control protocol with treatment decisions based on a threshold population abundance will be discussed. The benefits of the targeted use of pesticides will be clearly demonstrated using data on slug abundance collected in commercial fields.

We then argue that employing a single threshold for decision-making in the pesticide application protocol is not the most efficient way to assess risks associated with the population abundance when pesticide is applied selectively. It will be shown that a protocol for targeted use of pesticides depends heavily on the definition of a spatial density patch in heterogeneous spatial distribution, and a single decision-making parameter such as the population threshold cannot accommodate important information about the patch size. Hence an alternative is to introducetwo controlling parameters into the protocol in order to quantify the pest abundance in each patch and patch size separately and we discuss this approach in the paper.
\end{abstract}

Keywords discontinuous spatial distribution; sustainable pesticide use; pesticide reduction; slug patch;

\footnotetext{
${ }^{1}$ Corresponding author, email: n.b.petrovskaya@bham.ac.uk
} 


\section{Introduction}

Pesticide application is the most widely used means of pest control and it has been estimated that around $3 \times 10^{9} \mathrm{~kg}$ of pesticides are used across the globe per year Pimentel (2009). However, the indiscriminate use of pesticides can have serious negative consequences. Application of pesticides is costly and can risk damage to the environment Jepson et al. (1990). Pesticides are known to contribute to air, soil and water pollution DEFRA (2016) and there is also some evidence linking their use to human illnesses Alavanja et al. (2013); Pimentel and Greiner (1997). The overuse of pesticides can lead to insect resistance making future management a more difficult task Alyokhin et al. (2008). Finally, lethal or sub-lethal effects on non-target organisms such as natural enemies Sohrabi et al. (2013) can result in resurgence in the pest population or a secondary pest to emerge. Such risks are addressed by legislation governing the development and subsequent use of pesticidal products, and by technology that improves targeting and reduces drift, but there is a widespread recognition of the requirement to reduce and optimise the volumes used Matthews (2014, 2016).

Increased pressure to reduce the use of pesticides in agricultural crops results in an urgent need for new approaches to pest control that both reduce the number of applications in commercial agricultural fields and make those applications more precise. The concept of spatially targeted pesticide application to control pest population has already received the attention of researchers; e.g. see Archard etal. (2004); Brown et al. (2008); Pimentel (1997); Sotherton et al. (1993). Among other examples, the study in Brenner et al. (1998) has been focused on the probability of the presence of the pest in a spatial environment, allowing for the targeted use of pesticide in spatial areas where there is a high probability of pest presence. Probability mapping has also been done on larger scales in agriculture Fleischer et al. (1999) where sampling has been used to generate a probability threshold map, a contour map showing the probability of the number of pests within a known area exceeding a defined threshold. There have also been discussions on weed detection and targeted spraying of herbicide Miller (2003) as well as efforts to produce a system of automated robotic pesticide spraying over target areas for use in greenhouses Sammons et al. (2005). Most recently the concept of targeting molluscicide treatments at the spatially and temporally stable patches of higher slug densities that have been shown to occur in arable crops have been investigated in the field Forbes et al. (2017). Such studies, however, have not related targeted use of pesticides with the need to develop a monitoring and control protocol that takes account of the locations of spatial patches where the population density is high.

In our paper we develop a prototype protocol for targeted use of pesticides where the above issue is addressed. The grey field slug (Deroceras reticulatum (Muller, 1774)) has been selected as the baseline case to discuss optimal application of pesticide to strongly heterogeneous pest distributions and the issues that need to be resolved for successful targeting of pesticides in selected spatial domains. The grey field slug is an important pest of a wide range of agricultural and horticultural crops, resulting in significant economic losses in most years Nichols (2014); Twining et al. (2009). For many years slug control in arable crops has relied on molluscicide pellets applied to the entire field when the slug population exceeds defined thresholds. Mean- 
while, it has been reported in numerous studies Archard et al. (2004); Bohan et al. (2000); Forbes etal.(2017) that the spatio-temporal dynamics of the slug population results in heterogeneous spatial patterns of the slug density in arable fields whereby readily detected patches of higher slug numbers are interspersed within areas of lower slug densities irrespective of the population size. A patchy distribution of slugs may offer significant potential for reducing use of pesticides in agricultural fields. If a commercially viable method of identifying their location and size can be established then application of pesticides may be targeted at high slug density patches alone, leaving areas with lower slug numbers untreated.

Weexplain how to incorporatetargeted application of pesticides into a hypothetical protocol based on the threshold slug population abundance. The benefits of the targeted use ofpesticides will be clearly demonstrated when we investigate data on slug abundance collected in several commercial fields. The prototypeprotocol is sufficientlyflexible to be readily extended to other pest species that display a heterogeneous distribution. Meanwhile there remain several open questions that may constrain further development of the protocol, one of them being a definition of spatial patches. It will be argued in the paper that a generic definition of a patch as any spatial sub-domain with a closed boundary that has non-zero population density is not efficient when targeted use of pesticide is considered. Hence the generic definition of a spatial patch will be revisited to allow for the inclusion of various additional constraints on patch size and population density within the patch. We then investigate the impact of those constraints on the pesticide application procedure.

The paper is organised as follows. In Section 2 we introduce a generic targeted pesticide application procedure in a case where a population of grey field slugs has to be controlled. The section is focused on identification of areas with non-zero slug density with consequent application of pesticide in those areas, no matter how low the slug density is in each patch. In Section 3 we compare the results of Section 2 with a standard pesticide application protocol based on a threshold population abundance and demonstrate how the targeted application procedure should bemodified to takethethreshold numberinto accountand to avoidpesticide application in areas with low population density. We then show in Section 4 that a targeted use of the pesticide protocol depends heavily on the definition of a spatial patch and a single control parameter such as the population threshold in the current standard monitoring/control protocol cannot accommodate important information about patch size. An alternative to the 'single threshold' based approach is then to introduce two control parameters in order to quantify both the pest abundance in each patch and the patch size. Thus, we explain in Section 4 how to take into account the pest density and the patch size in the pesticide application protocol. Finally, conclusions and directions for future work are considered in Section 5 .

\section{The baseline method}

Our aim in this section is to explain a hypothetical pesticide application protocol that can be considered as a prototype for targeted use of pesticide. In this and the next section we 
demonstrate our approach by using the baseline case of the grey field slug population.

The grey field slug (Deroceras reticulatum) is a gastropod mollusc that is widespread in Europe and in particular in the UK where the data we use in our study havebeen collected. Theslug is a slowmoving species and hastwoactivity peaks in arablefields, between November and December and again between late-February and May Port and Port (1986). Data on slug abundance were collected by researchers in several commercial winter wheat fields between autumn 2015 and autumn 2017 Forbes et al. (2017). A standard experimental design was established for the research in all fields in both cropping years. Refuge traps consisting of upturned $18 \mathrm{~cm}$ diameter, plastic plant pot saucers were placed in a regular $10 \times 10$ grid with distance $h=10$ meters between nearest traps. Sampling grids were installed at a minimum of 20 meters from the nearest field edge. The number of slugs under each refuge trap were recorded after traps had been left undisturbed for 14 days, and thereafter at regular intervals.

An important assumption in our study was that the sampling grid used for collection of slug data was sufficiently fine to provide accurate information about both the heterogeneous spatial slug distribution and the total slug abundance in the field. We would like to emphasize here that although the above assumption is essential for our discussion, we recognise that using such a fine sampling grid (i.e. 100 traps per hectare) is not realistic in routine data collection for commercial pest management. Determining a minimum number of sampling locations required for accurate reconstruction of spatial density patches is a challenging question that requires further careful study. While the above question is beyond a scope of this paper, it will be a topic of future work.

The dataset we use to introduce our approach is a spatial distribution of the slug population obtained from trap counts collected on 18 November 2016 from a commercial arable field sown with winter wheat, located at South Kyme, Lincolnshire, UK. The trap count data collected in the field are shown in Table 1, and the spatial slug distribution reconstructed from these data is shown in Fig. 1 where the total length $L$ of the sampling grid $(100 \mathrm{~m})$ in both directions is converted to the non-dimensional unit length $L=1$; see Appendix A for further explanation of the conversion of the GPS coordinates in the physical domain to the grid coordinates in the unit square. Given that a regular grid of traps is used in the monitoring routine, the distance $h$ between traps and the coordinates of each trap are readily calculated in the converted domain. The continuous distribution shown in the figure has been obtained from the discrete data in Table 1 by using the MATLAB software MATLAB (2017). An interpolation function 'interp2' in MATLAB takes trap counts that are available at trap locations only and converts that data into a continuous population density function defined at every point of the domain of interest by applying piecewise linear interpolation. Slug patch is then defined as any spatial sub-domain with the closed boundary where the population density is greater than zero (cf. Fig.1a).

For the sake of discussion in this section we assume that a pesticide will be applied in our hypothetical protocol to any slug patch in the field, no matter how low the slug density is within the patch. Thus the first step in our procedure is to identify the boundaries of spatial patches that are clearly seen in Fig. 1a. Employing the MATLAB software for this purpose requires converting the data in Table 1 into binary data (i.e. producing the presence/absence 


\begin{tabular}{|l|l|l|l|l|l|l|l|l|l|}
\hline $\mathbf{1}$ & 0 & 0 & $\mathbf{1}$ & 0 & $\mathbf{2}$ & 0 & 0 & 3 & 0 \\
\hline 4 & $\mathbf{1}$ & 0 & 0 & 0 & 0 & 0 & $\mathbf{1}$ & 0 & 0 \\
\hline 0 & 0 & 0 & 0 & 0 & $\mathbf{1}$ & 0 & 0 & 0 & 0 \\
\hline 0 & 0 & 0 & 0 & $\mathbf{1}$ & $\mathbf{1}$ & 0 & $\mathbf{1}$ & 0 & $\mathbf{1}$ \\
\hline $\mathbf{1}$ & $\mathbf{2}$ & 0 & $\mathbf{1}$ & 0 & $\mathbf{1}$ & 0 & 0 & 0 & 0 \\
\hline $\mathbf{2}$ & $\mathbf{2}$ & $\mathbf{1}$ & 0 & 0 & 0 & 0 & 0 & 0 & 0 \\
\hline 0 & $\mathbf{1}$ & $\mathbf{1}$ & 0 & 0 & 0 & $\mathbf{1}$ & $\mathbf{1}$ & 0 & 0 \\
\hline 0 & 0 & 0 & 0 & 0 & 0 & 0 & 0 & 0 & $\mathbf{1}$ \\
\hline 0 & 0 & 0 & 0 & 0 & 0 & $\mathbf{1}$ & $\mathbf{2}$ & 0 & $\mathbf{1}$ \\
\hline 0 & $\mathbf{1}$ & 0 & 0 & $\mathbf{1}$ & 0 & 0 & 0 & 0 & 0 \\
\hline
\end{tabular}

Table 1: Trap count values taken from a regular sampling grid of $10 \times 10$ traps in the South Kyme field on 18 November 2016.

map from the original data). Consider trap count $C_{i}$ in the $i$ th refuge trap $i=1,2, \cdots, N$, where $N$ is the number of traps in the field, and let $h$ be the distance between neighboring grid points on a regular sampling grid. For each data point, if the trap count is $C_{i}>$ o then we set $C_{i}=1$ and if $C_{i}=\mathrm{o}$ it remains set as o. This produces a binary table showing only the location of slugs (as linked to the position of each trap in the field) and not the quantity. Neighbouring values of 1 in the horizontal or vertical direction are then said to be in the same patch and we can therefore count distinct patches in the field and define their boundaries by using the image processing package in MATLAB (2017).

The patch boundaries are visualised in Fig. 1b. We assume that every trap is installed at the centre of its square catchment area and the catchment area has the linear size of $h / 2$, where $h$ is the distance between neighboring traps. Hence a slug patch which appears as an isolated non-zero trap count in Table 1 will have the same area as trap catchment area $A=h \times h$. That assumption is illustrated in Fig. 1b where the regions shown in green within slug patch are regions enclosed by neighbouring traps that contain slugs and the outer region shown in blue has the width of $h / 2$. Hence, in patches shown purely in blue, there are only one or two traps where slugs were found and their neighboring traps did not contain slugs.

We now model the application of pesticide so that the entire area of a patch is covered. The pesticide is applied when the spreader is moved along a track in the field and in our model we assume that the field has three interior tracks that are shown as solid vertical lines in Fig. 1c. Each vertical track in the figure is completely defined by its $\mathrm{x}$-coordinate, $x^{t}$, and we then label the tracks as $x^{t}, x^{t}$ and $x^{t}$ respectively. We also assume that two external tracks (i.e. vertical boundaries $x^{1}=2$ and $x^{3}=1$ labeled as $x_{1}^{e}$ and $x_{2}^{e}$ in Fig. 1c) can be used for pesticide spreading, the pesticide can be spread in either direction from a track (i.e. to the left and to the right), and the pesticide is uniformly distributed between the track and the midpoint between two tracks. Therefore if a patch on one side of the track is detected, pesticide will be spread in a rectangular block.

The track lines and the midpoints between tracks are represented in Fig. 1b-d as solid and dotted lines respectively. In our algorithm we define projection of each slug patch onto track 
a
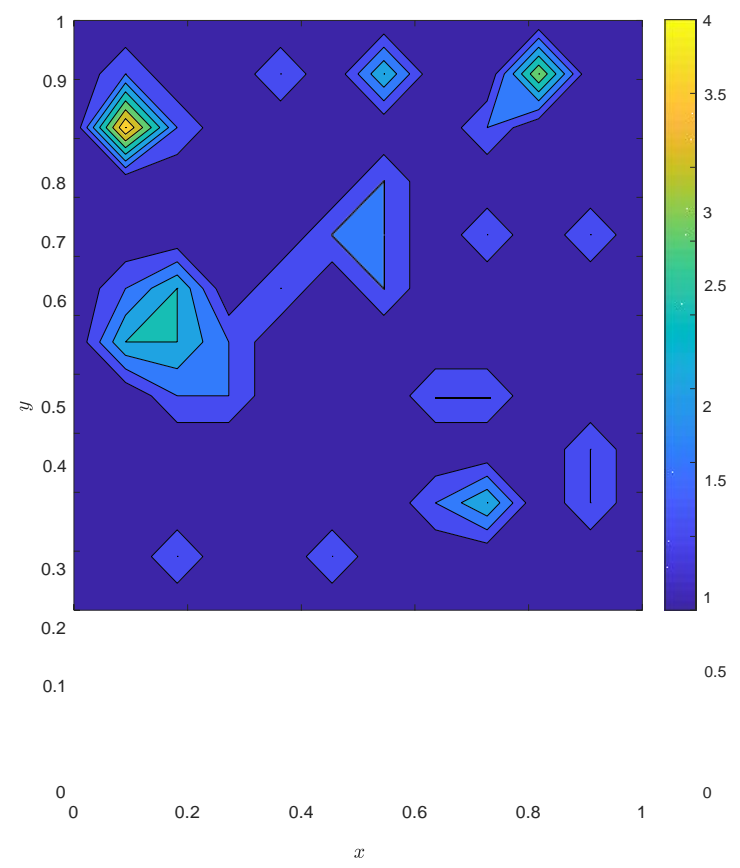

$\mathrm{c}$

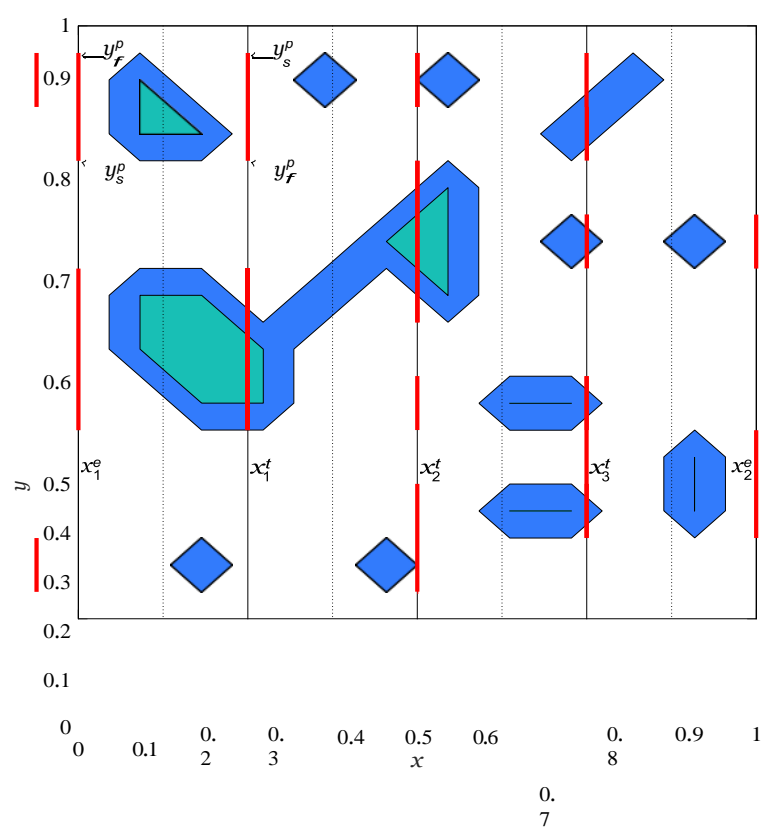

$\mathrm{b}$
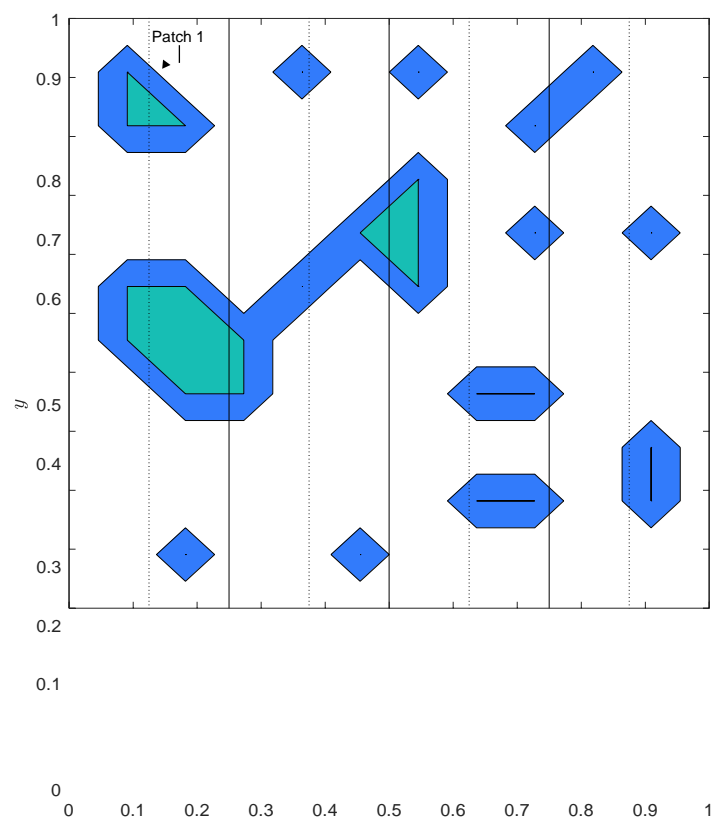

d

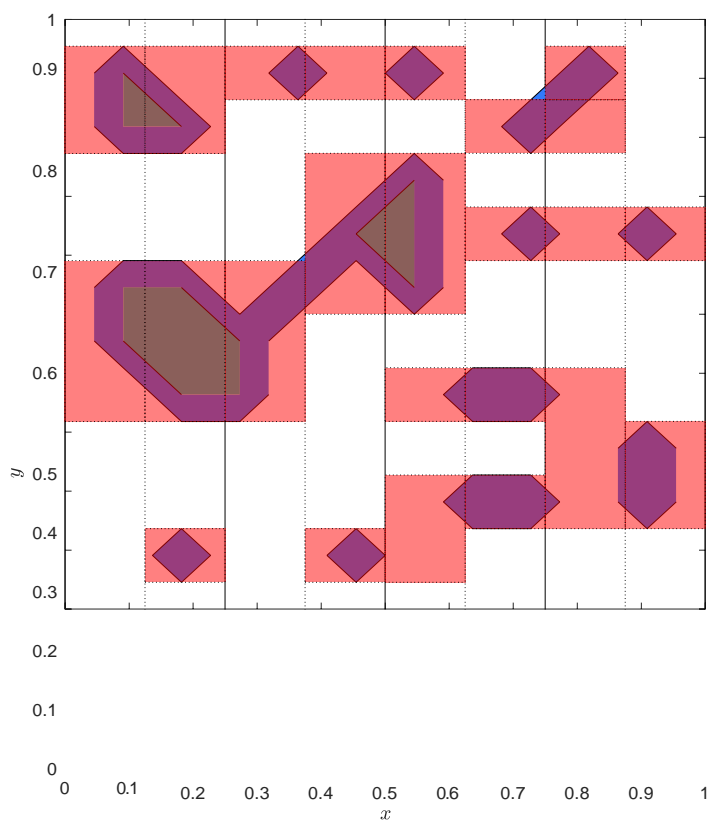

Figure 1: Trap counts were taken on a sampling grid of 10 by 10 locations in the South Kyme field on 18 November 2016 (see details in the text), the corresponding numerical values are given in Table 1. The total length $L$ of the sampling grid $(100 \mathrm{~m})$ in the $x$ and $y$ directions is rescaled as $L=$ 1. The continuous distributions and patch boundaries shown in the figure are obtained by using the MATLAB software. (a) The slug spatial distribution reconstructed from trap counts based on linear 
interpolation between trap locations. (b) Contour plot showing slug patches (areas of light green colour in the figure) with the boundary region (blue colour). (c) Contour plot showing slug patches mapped onto the tracks. The mappings are shown as red lines along the track indicating the points from where pesticide should be applied. (d) Contour plot showing slug patches with pesticide applied in red shaded areas. Pesticide used $M=45.5 \%$. 
lines and those coordinates are used to generate rectangular domains where pesticide will be applied. For each slug patch $p$ to be handled, the algorithm outputs the track label (as explained above) and the coordinates $y_{s}^{p}$ and $y_{f}^{p}$ along the track where pesticide should start being applied and stop being applied.

Consider, for example, the first slug patch labeled as 'patch 1' at the upper left corner of Fig. 1b. In order to cover that patch with pesticide, it should be applied when the spreader moves from point $y_{s}^{p}$ to $y_{f}^{p}$ along track $x_{1}^{e}$ and when it then moves in the opposite direction from point $y_{s}^{p}$ to $y_{f}^{p}$ along track $x_{i}^{t}$; see Fig. 1c. The above information is recorded, is stored along with the patch label, and the same analysis is made for any slug patch detected in the field. As a result, the algorithm returns all data required for targeted use of pesticides in the field. The output data are illustrated in Fig. 1c: spreading of pesticide will happen when the spreader moves along each interval shown in red.

Once the output information has been obtained, the rectangular domains are restored to contain each patch where the pesticide should be spread. The areas of the field where pesticide is applied can be seen by the shaded areas in Fig. 1d. The total area $A$ of pesticide applied to the field is calculated by summing the area of each shaded rectangular block. Since the total field area is $A_{*}=L \times L=1$ and assuming that total coverage of the field would use $M_{*}=$ (D mass units of pesticide (i.e. $100 \%$ of pesticide), we can find the percentage of pesticide $M$ used by targeting only the patches as $M=A \times M_{*}$. In the case of South Kyme, when the patches are targeted from three internal tracks and two external tracks, only $M=45.5 \%$ of pesticide is used.

\section{Threshold-based pesticide application}

The example of the South Kyme field in the previous section is convenient for the purpose of illustration of our approach as it presents a patchy spatial distribution with very distinct slug patches. However, if the data in Table 1 were considered under more realistic conditions, then no pesticide would be used at all. Monitoring and control protocols usually require that a management action is only applied if an estimate of the average trap count (or the average density) exceeds agiven management threshold Stern etal.(1959). Althoughindividuals from different slug life stages are unlikely to have the same negative impact on crops, average trap count in this study reflected normal commercial practice in referring to total trap count (adult + sub-adult + juvenile slugs). Let us define average trap count $S$ as

$$
S={\underset{N}{i=1}}_{C_{i},}^{N}
$$

e.g. see Davis (1994); Snedecor and Cochran (1980) where $C_{i}$ is the trap count in the $i$ th refuge trap and $N$ is the number of traps in the field. In most pesticide application protocols average trap number $S$ has to be checked against the imposed action threshold value $S_{t h}$ and pesticide will only be applied if $S \geq S_{t h}$. Let us define a threshold value in our hypothetical protocol as $S_{t h}=5$ (note this is higher than the usual thresholds used in commercial practice 


\begin{tabular}{|l|l|l|l|l|}
\hline Field & Date & $P$ & $M \%$ & $S$ \\
\hline Uppington & 04.01 .16 & 2 & 100 & 1.78 \\
Uppington & 19.01 .16 & 4 & 100 & 1.29 \\
Uppington & 01.02 .16 & 5 & 92.5 & 1.65 \\
Uppington & 16.02 .16 & 12 & 32.5 & 0.2 \\
Adney Corner & 30.11 .15 & 13 & 35.0 & 0.25 \\
Adney Corner & 14.12 .15 & 14 & 77.5 & 0.69 \\
Adney Corner & 29.01 .16 & 3 & 100 & 0.98 \\
Adney Corner & 16.02 .16 & 18 & 55 & 0.42 \\
Stoney Lawn & 07.12 .15 & 1 & 100 & 3.02 \\
Stoney Lawn & 06.01 .16 & 1 & 100 & 5.98 \\
Stoney Lawn & 11.01 .16 & 1 & 100 & 6.21 \\
Stoney Lawn & 14.01 .16 & 2 & 100 & 6.03 \\
Stoney Lawn & 18.02 .16 & 1 & 100 & 9.13 \\
Badjics & 18.12 .15 & 5 & 91.3 & 1.61 \\
Badjics & 06.01 .16 & 2 & 100 & 2.24 \\
Badjics & 20.01 .16 & 2 & 100 & 1.60 \\
Badjics & 18.02 .16 & 1 & 100 & 4.00 \\
Adney Middle & 08.12 .15 & 4 & 100 & 1.20 \\
Adney Middle & 22.12 .15 & 1 & 100 & 1.68 \\
Adney Middle & 14.01 .16 & 1 & 100 & 2.28 \\
Adney Middle & 18.01 .16 & 1 & 100 & 4.00 \\
Adney Middle & 12.02 .16 & 1 & 100 & 2.35 \\
\hline
\end{tabular}

Table 2: Analysis of trap count data collected on several commercial fields. The number of patches $P$ is defined by the MATLAB software where slug patch is considered as an isolated subdomain with the non-zero slug density (see details in the text). The average trap count $S$ is calculated as in (1). The amount of pesticide $M$ needed to cover all patches in the field is calculated as the percentage of the amount needed to cover the entire field.

which can be up to a mean of 4 slugs per trap depending on the crop or crop condition being treated). In our model case of the South Kyme field the average trap count is $S=0.49$ and is much less than $S_{t h}$. Given a very low number of slugs, there is no need to apply pesticide.

The above conclusion about the use of pesticide is further confirmed by data in Table 2 where we show the result of our algorithm applied to spatial distribution of slugs in several commercial fields. Again we assume that total coverage of the field would use $M=100$ mass units of pesticide. The analysis of the amount of pesticide $M$ (column 4 in the table) reveals that in most cases the entire field will be covered by pesticide if we aim to control all slug patches without taking the threshold value into account. On the other hand, it is seen from the table that the average trap count $S$ in column 5 is well below the threshold value for many commercial arable fields presented in the table and pesticide application is not requiredin 


\begin{tabular}{|l|l|l|l|l|l|l|l|l|l|}
\hline $\mathbf{1 1}$ & 0 & $\mathbf{2}$ & 0 & 5 & 7 & 9 & $\mathbf{1}$ & $\mathbf{1}$ & 7 \\
\hline $\mathbf{2 1}$ & $\mathbf{1}$ & 0 & 3 & $\mathbf{1}$ & 3 & $\mathbf{1 5}$ & 3 & 7 & 0 \\
\hline 10 & 0 & 6 & $\mathbf{2}$ & $\mathbf{2}$ & 0 & 8 & 0 & 3 & 4 \\
\hline 16 & 0 & 2 & 2 & $\mathbf{1}$ & 2 & $\mathbf{2 3}$ & 2 & 5 & 3 \\
\hline $\mathbf{1}$ & 6 & 0 & 2 & 4 & 7 & $\mathbf{1 8}$ & 4 & 4 & 0 \\
\hline 18 & 0 & $\mathbf{1}$ & 2 & $\mathbf{1 4}$ & $\mathbf{1 2}$ & $\mathbf{1 0}$ & 2 & 5 & 0 \\
\hline 32 & 5 & 3 & 2 & 5 & $\mathbf{1}$ & 34 & 6 & 6 & 0 \\
\hline 15 & 3 & $\mathbf{1}$ & 0 & $\mathbf{1 2}$ & $\mathbf{2}$ & 48 & 4 & 8 & $\mathbf{2}$ \\
\hline 2 & 3 & 0 & 4 & $\mathbf{1 0}$ & 4 & $\mathbf{2 0}$ & 3 & 2 & $\mathbf{1}$ \\
\hline $\mathbf{1}$ & $\mathbf{1 7}$ & 7 & 9 & 3 & 4 & $\mathbf{1 7}$ & 3 & 4 & $\mathbf{2}$ \\
\hline
\end{tabular}

Table 3: Trap count values from the $10 \times 10$ sampling grid in the Stoney Lawn field. Trap counts were taken on 14 January 2016.

those fields at all. We conclude from comparison of columns 3, 4 and 5 that in most cases presented in the table we have a small number of slug patches (column 3 ) and the slug density is low within each patch. Hence our next step is to incorporate the threshold criterion in our algorithm in order to avoid application of pesticide to slug patches with low slug density.

To explain our approach, let us consider another example where slug data were collected in a field in Stoney Lawn, Shropshire, UK, on 14 January 2016. The main difference between this dataset and the South Kyme dataset considered previously is that there is a much larger slug population in Stoney Lawn. Table 3 shows the trap count values where the values range from o to 48 with average $S=6.03$ and we also note that the majority of trap counts are at least 1.

The spatial slug distribution reconstructed from the data in Table 3 is shown in Fig. 2a. The patchidentification procedure explained in Section 2 is then applied and patch boundaries are shown in Fig. 2b where we can see one very large patch covering the majority of the field. Therefore, if we were to apply the algorithm as in the previous example of the South Kyme field, almost the entire field would need to be targeted. Meanwhile, visual inspection of spatial slug distribution in Fig. 2a reveals that there are several areas of the low slug density where pesticide is not required along with one area of very high density where the pesticide must be applied. Let us recall that in the targeted use of pesticide procedure in Section 2 we have defined slug patch as any sub-domain of the non-zero slug density in the field and that simplistic definition is not efficient when the density of slugs varies in different patches. A more careful definition of patches is therefore required to allow for selection of patches with the high density and that should be incorporated into the targeted use of pesticide protocol.

\subsection{The threshold-based protocol}

When a pesticide is applied uniformly across the whole field, threshold $S_{t h}$ is a key parameter in the monitoring and control protocol as it determines whether pesticide application is required or not. Therefore, one method of choosing the patches to target would be to list the traps in 


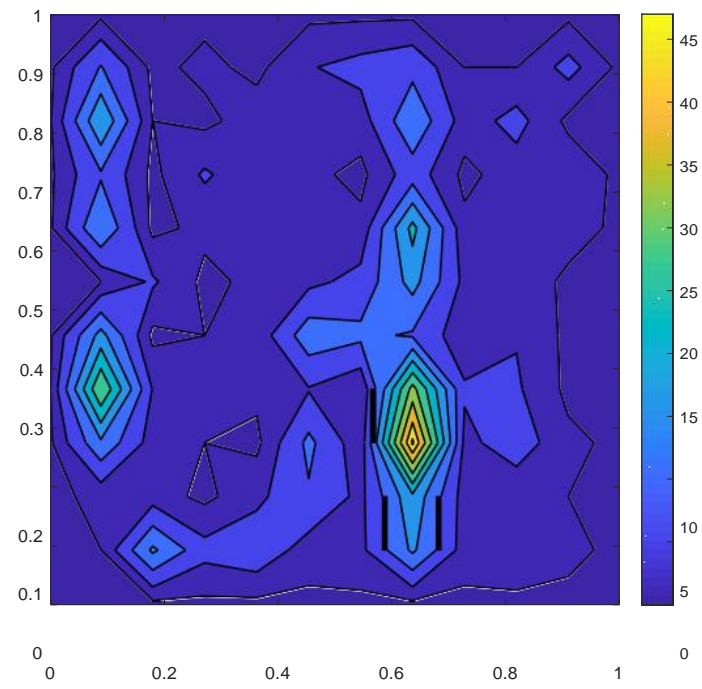

c

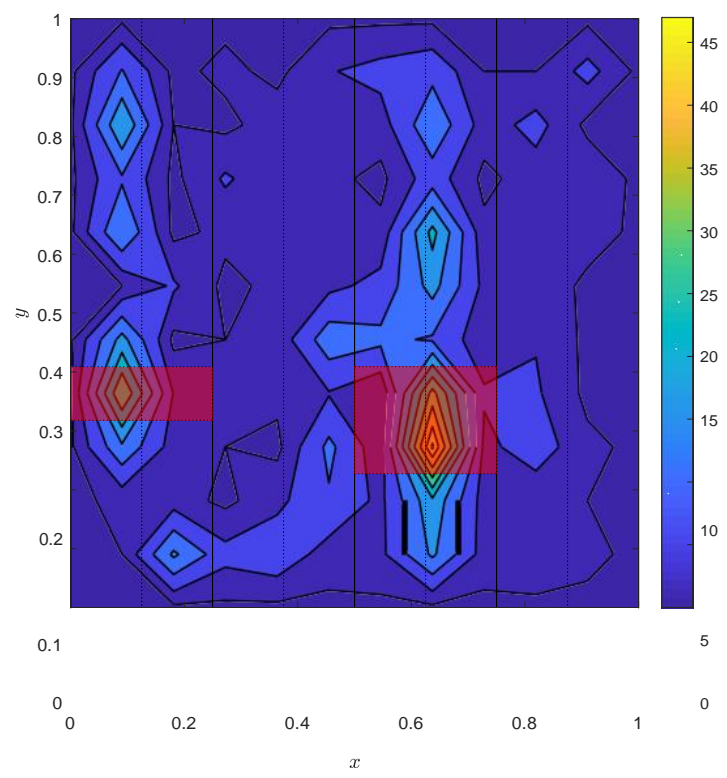

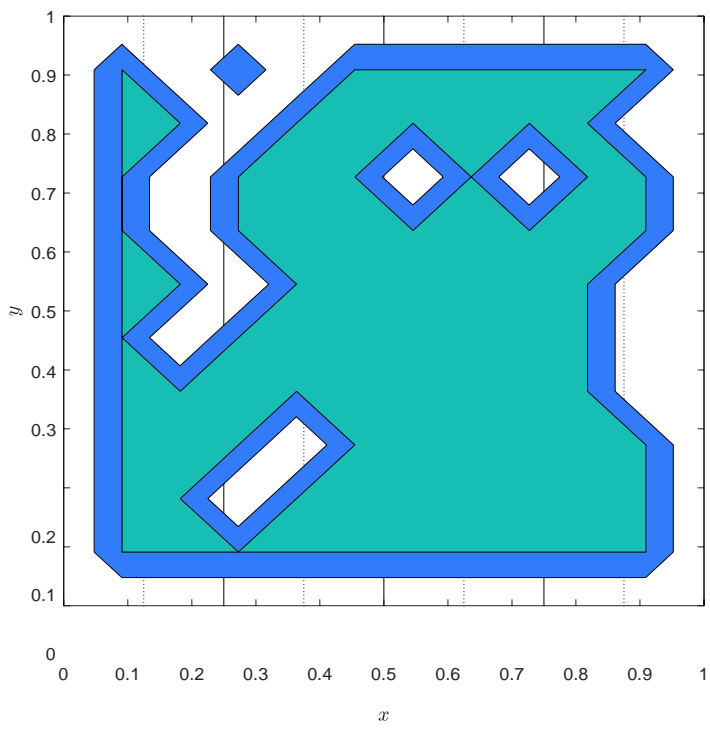

d

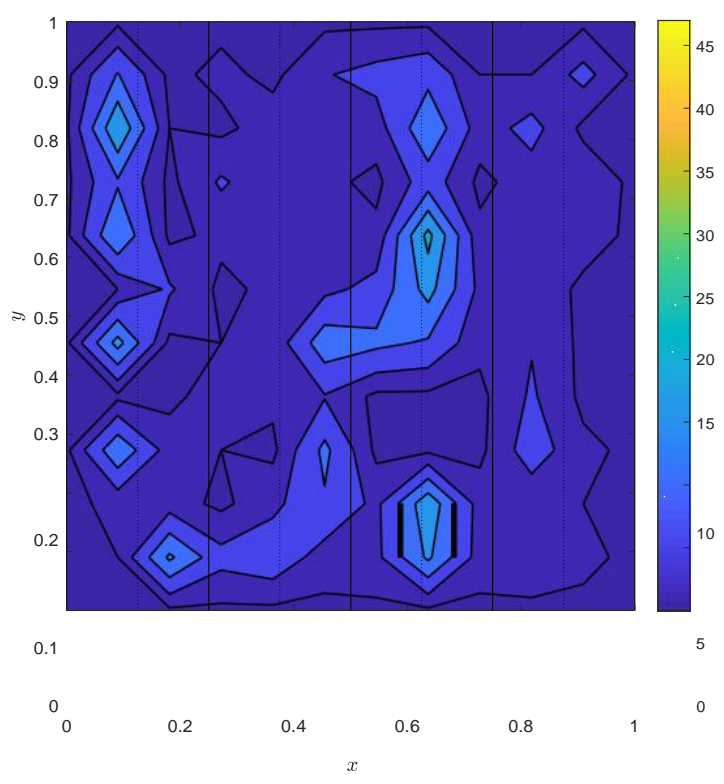

Figure 2: Trap counts were taken on a regular sampling grid of 10 by 10 locations in the Stoney Lawn field on 14 January 2016 (see also Table 3). The total length L of the sampling grid in the $x$ and $y$ directions is rescaled as $L=1$. The continuous distributions of the slug density in the field shown in the figures were obtained from the discrete data by using MATLAB software. (a) The slug spatial distribution reconstructed from trap counts in Table

3. (b) Contour plot showing areas with the non-zero slug density at Stoney Lawn. (c)-(d) Application of the thresholding procedure with a threshold of $S_{t h}=5$. (c) Two patches with the highest slug density are identified and pesticide will be applied in red shaded areas only.

(d) The distribution of slugs after the pesticide has been applied. The field has an original average trap count of $S=6.03$. After the application of pesticide, the new average trap count 
is $S=4.71$. The amount of pesticide used was $7.5 \%$ of that which would be used to cover the entire field (cf. Fig. 2b). 
descending order of the size of the trap count. The average trap count $S$ is then calculated according to (1). If the average trap count is above the threshold $S_{t h}$, the trap with the maximum trap count is marked to be targeted and the area of potential pesticide application is defined. The average trap count $S$ is then recalculated under the assumption that the trap already marked to be targeted now has a zero trap count. If the new average trap count in (1) is still above the threshold then the trap with maximum remaining trap count is also marked to be targeted. The average trap count $S$ is again recalculated with any traps that have been targeted set to zero along with any other traps in the area of pesticide application. The above procedure is repeated until the recalculated average value $S$ is below the threshold or below the targeted pest suppression; see Appendix B for more detail.

One important observation which should be incorporated into the procedure is that the pellet applicator is restricted to moving along defined tracks applying pellets to a swathe extending outwards on either side. Because of those restrictions when pesticide is applied to a targeted area around a trap, other traps within that area may have pesticide applied to them irrespective of the trap count(i.e. despite not being specifically marked out to be targeted). We therefore have to set counts from such traps to zero before the next iteration of the procedure.

Returning to the example of the field in Stoney Lawn, using the above procedure requires the areas around only three traps to be targeted before an average value $S<S_{t h}=5$ is obtained. The average of all trap counts in Stoney Lawn is $S=6.03$ but after targeting the traps with values 48,34 , and 32 (the three highest counts as seen in Table 3 ) the average is reduced to $S=4.71$. The patches of high slug density to be targeted are shown in Fig. 2c.

As explained above, after the pesticide has been applied, we assume that any trap within the area targeted by pesticide has a zero trap count. In the Stoney Lawn field three traps were marked for pesticide treatment, but an additional four were also within the swathe in which pellets were applied when the spreader moved along each track. Hence we have seven traps in total where the trap count is set to zero after pesticide application. Those 'new' zerotrap counts are taken into account in formula (1) when the average is calculated after pesticide application. The trap counts in the traps that have pesticide applied account for $22.9 \%$ of the total number of slugs in the field ( 132 out of 603) but the pesticide used is now only $M=7.5 \%$ of the amount required to cover the entire field. The resulting hypothetical distribution of slugs after the pesticide has been applied is shown in Fig. 2d.

If we use the same example of the Stoney Lawn field but change the threshold to $S_{t h}=3$, a higher number of traps will be required to be targeted to reduce the average $S$ below the threshold. In this case, 10 traps are chosen for targeting with 23 traps having pesticide applied in total. Fig. 3 shows the outline of the patches to be targeted in relation to the distribution of slugs. The average slug count from the remaining traps is reduced to $S=2.91$. The trap counts set to zero after pesticide application account for $51.7 \%$ of the total population (i.e. 312 out of 603 slugs) and the amount of pesticide used is $26.2 \%$. Conversely, if the threshold $S_{t h}$ is increased then fewer traps will be targeted. If $S_{t h}=6$ then only one trap with the trap count of 48 will be targeted and if $S_{t h}=7$ then there will beno pesticide applied in the field as the average trap count $S=6.03$ is already below the threshold. 


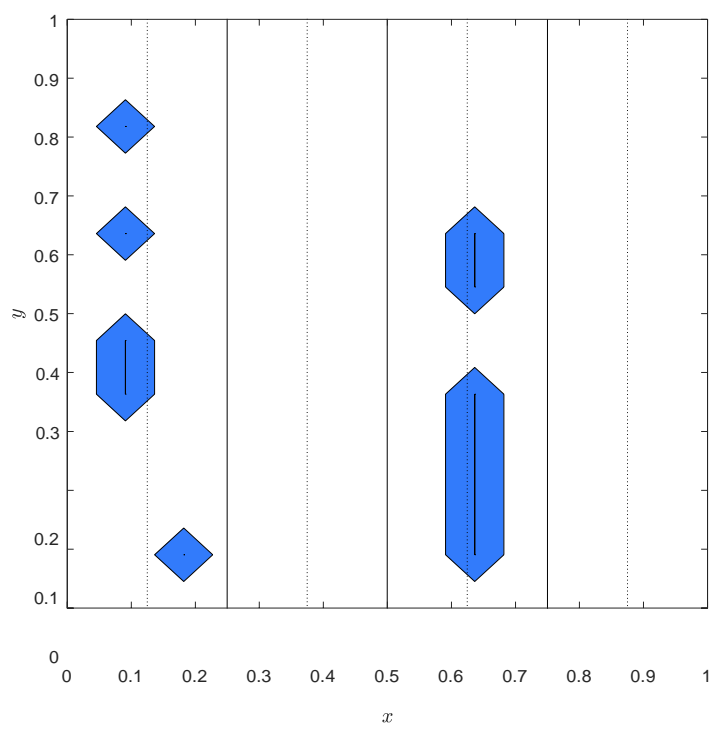

$\mathrm{b}$

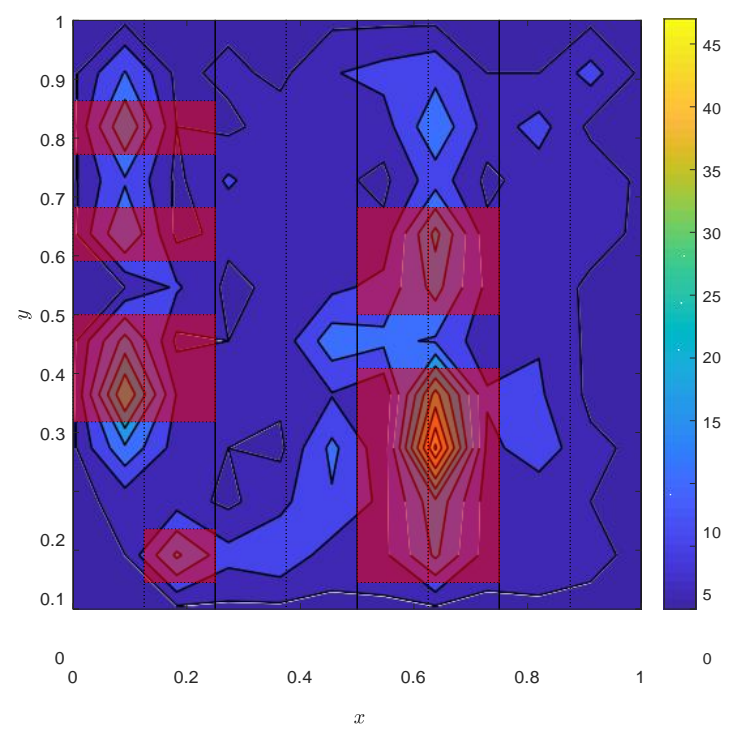

Figure 3: Targeted application of pesticide in the Stoney Lawn field with a threshold of $S_{\text {th }}=3$. (a) Contour plot showing slug patches to be targeted. (b) The spatial distribution reconstructed from data in Table 3) with pesticide to be applied in red shaded areas after slug patches to be targeted have been identified. The field has an average trap count $S=6.03$ and after the application of pesticide the new average trap count is $S=2.91$. The amount of pesticide used is now $26.2 \%$ of what would be used to cover the entirefield.

\begin{tabular}{|l|l|l|l|l|l|}
\hline Field & Date & $S$ & $S_{\text {new }}$ & $M \%$ & $C_{\text {max }}$ \\
\hline Stoney Lawn & 06.01.16 & 5.98 & 3.97 & 22.50 & 11 \\
Stoney Lawn & $\mathbf{1 1 . 0 1 . 1 6}$ & 6.21 & 3.76 & 23.75 & 13 \\
Stoney Lawn & $\mathbf{1 4 . 0 1 . 1 6}$ & 6.03 & 3.95 & 15.00 & 18 \\
Stoney Lawn & $\mathbf{1 8 . 0 2 . 1 6}$ & 9.13 & 3.51 & 41.25 & 12 \\
Badjics & $\mathbf{1 8 . 0 2 . 1 6}$ & 4.00 & 3.78 & 2.50 & $\mathbf{1 1}$ \\
Adney Middle & $\mathbf{1 8 . 0 1 . 1 6}$ & 4.00 & 3.81 & 1.25 & $\mathbf{1 1}$ \\
\hline
\end{tabular}

Table 4: The results of targeted pesticide application based on the threshold value $S_{\text {th }}=4$. The average trap count is calculated before $(S)$ and after $\left(S_{n e w}\right)$ pesticide application; see details in the text. The amount of pesticide $M$ needed to cover marked areas in the field is calculated as the percentage of the amount of pesticide needed to cover the entire field. $C_{\text {max }}$ is the highest remaining trap count after pesticide application.

\subsection{Targeting patches with high slug density: examples}

We now investigate the threshold-based protocol in more detail by employing it for decision making on pesticide application in several fields in Shropshire which have relatively high average trap count $S \geq 4$ (Table 2). Spatial distributions of slugs in those fields are shown in Fig. 4 and patches with high slug density are clearly visible in each field. We assume that those fields are subject to pesticide application as they exceed a threshold value $S_{t h}=4$. The 


\begin{tabular}{|l|l|l|l|l|l|}
\hline Field & Date & $S$ & $S_{\text {new }}$ & $M \%$ & $C_{\max }$ \\
\hline Stoney Lawn & 06.01.16 & 5.98 & 3.64 & 24.00 & 10 \\
Stoney Lawn & 11.01 .16 & 6.21 & 3.91 & 19.00 & 13 \\
Stoney Lawn & 14.01 .16 & 6.03 & 3.67 & 16.00 & 17 \\
Stoney Lawn & 18.02 .16 & 9.13 & 3.80 & 38.00 & 12 \\
Badjics & 18.02 .16 & 4.00 & 3.78 & 2.00 & 11 \\
Adney Middle & 18.01 .16 & 4.00 & 3.81 & 1.00 & 11 \\
\hline
\end{tabular}

Table 5: The results of targeted use of pesticide when the number of interior tracks is increased from 3 (see previous examples) to 4. The threshold value is $S_{\text {th }}=4$. The legend is as in Table 4.

results of pesticide application are shown in Table 4. The average trap count $S$ calculated for original spatial distribution in each field is compared with the new average trap count $S_{n e w}$ recalculated after pesticide application. It is seen from the table that $S_{\text {new }}$ is below the threshold value in all fields. Let us notice, however, that the heterogeneous spatial distribution remains and slug patches are visible in all fields after pesticide application (Fig. 4). Only a fraction of the total population is treated as a result of the threshold-based protocol and in several cases just one or two patches should be removed in order to meet the condition $S \leq S_{t h}$.

One example of very small pesticide usage is given by the Badjics field where it is sufficient to treat only one of the patches with higher population density to reduce the overall population level in the area studied to below threshold level (cf. Fig. 4e(i) and e(ii) where the spatial distribution is shown before and after pesticide application respectively). It requires $M=$ $1.25 \%$ to treat the patch and move the average trap count at the Badjics field below the threshold. However, despite the condition $S \leq S_{t h}$ being formally fulfilled, the new average trap count $S=3.81$ remain very close to the threshold. It can be seen from the spatial distribution in Fig. 4e(ii) that the slug patches still have relatively high population density. Hence a more careful definition of the pesticide application protocol may be required, and we discuss an alternative approach in the next section.

Let us now investigate what happens when we change the number of tracks used by the pellet applicator when moving across the field. The results are shown in Table 5 where the number of interior tracks is increased from 3 (as in previous examples) to 4 . We anticipate

\begin{tabular}{|l|l|l|l|l|}
\hline Field & Date & $M \%\left(S_{t h}=3\right)$ & $M \%\left(S_{t h}=4\right)$ & $M \%\left(S_{t h}=5\right)$ \\
\hline Stoney Lawn & 06.01 .16 & 40.00 & 22.50 & 12.50 \\
Stoney Lawn & 11.01 .16 & 33.75 & 23.75 & 12.50 \\
Stoney Lawn & 14.01 .16 & 26.25 & 15.00 & 7.50 \\
Stoney Lawn & 18.02 .16 & 50.00 & 41.25 & 26.25 \\
Badjics & 18.02 .16 & 13.75 & 2.50 & 0 \\
Adney Middle & 18.01 .16 & 12.50 & 1.25 & 0 \\
\hline
\end{tabular}

Table 6: The results of targeted use of pesticide when the threshold number $S_{\text {th }}$ varies (see the threshold value $S_{\text {th }}$ in brackets). The legend is as in Table 4. 
a(i)

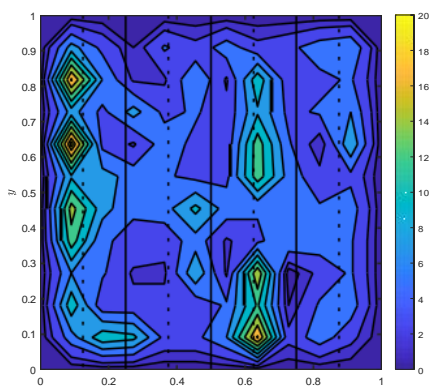

a(ii)

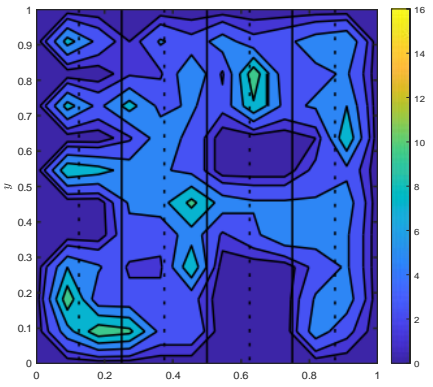

d(i)

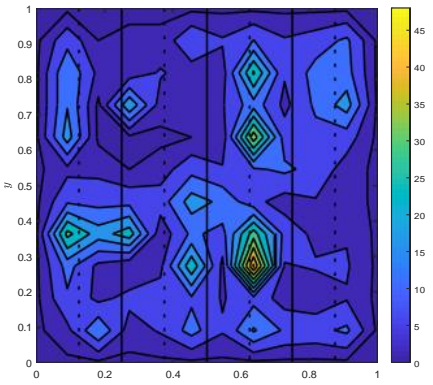

d(ii)

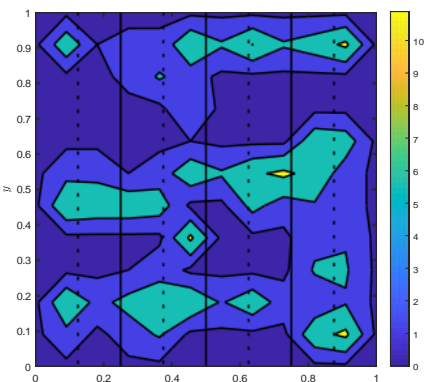

b(i)

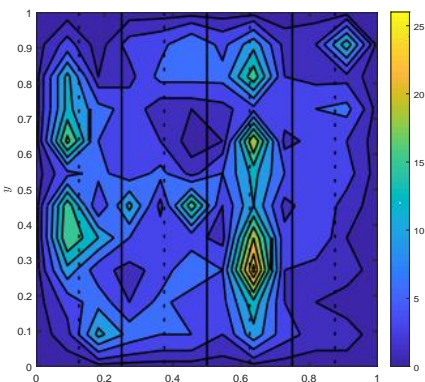

b(ii)

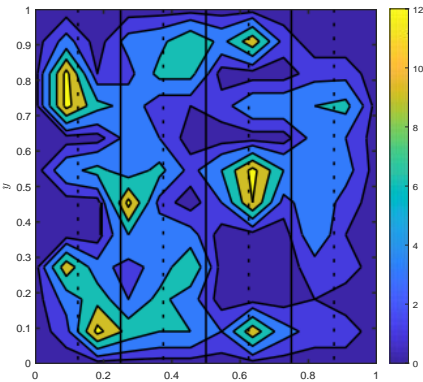

e(i)

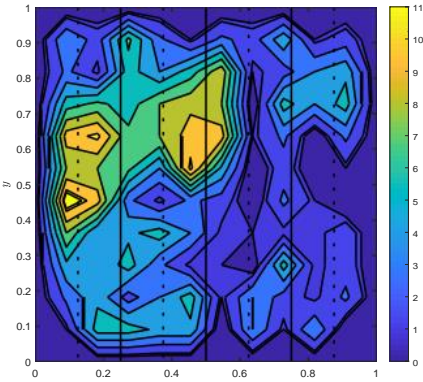

e(ii)

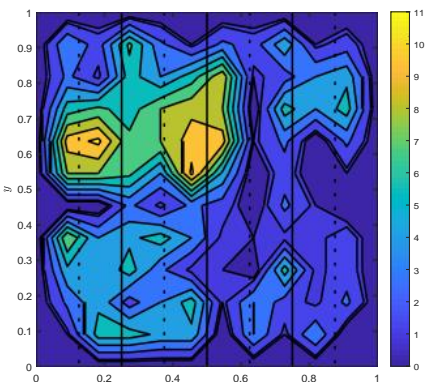

c(i)

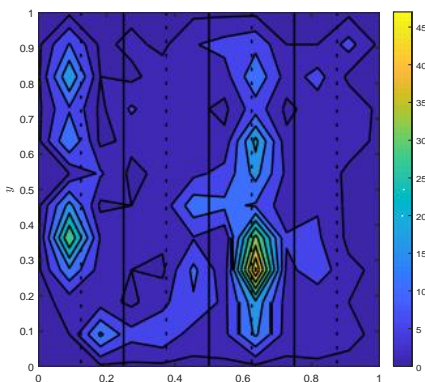

c(ii)

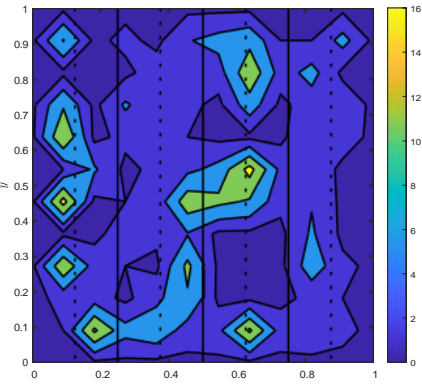

f(i)

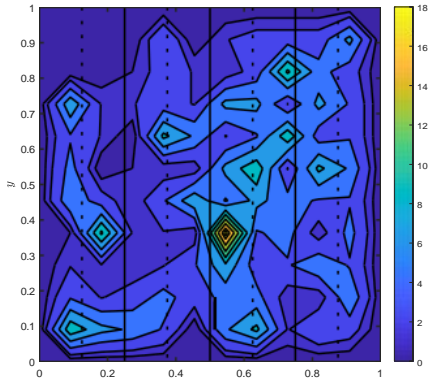

f(ii)

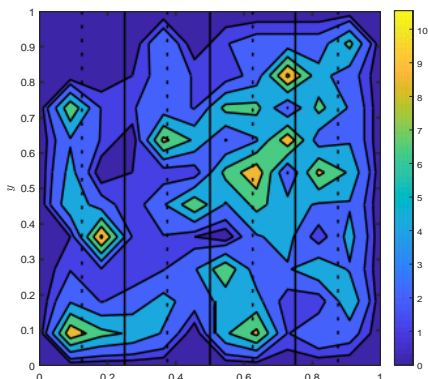

Figure 4: The spatial distribution of slugs from trap counts (i) before and (ii) after pesticide has 
been applied to targeted traps. The action threshold is $S_{t h}=4 .(a)-(d)$ represent spatial distributions at the Stoney Lawn field on 06.01.2016 (a), 11.01.2016 (b), 14.01.2016 (c), and 8.02.2016 (d), (e) the Badjics field on 18.02.2016 and $(f)$ the Adney Middle field on 18.01.2016. 
that if we were to increase the number of tracks in the field, we would be able to target the patches with a smaller total area more accurately and fewer traps that are not targeted will have pesticide applied to them. However, the results presented in Table 5 do not justify using extra tracks for more efficient pesticide application. It is seen from the table that the amount of pesticide used in each field remains approximately the same (or even increases in some cases) when another track is added to the route. Thus the optimal number of tracks in the field remains an open question and development of a reliable protocol with regard to this issue will require further study.

Finally, we investigate how the pesticide consumption depends on the threshold value employed in the protocol. We anticipate from our discussion in Section 3.1 that changing the threshold value will change the amount of pesticide used on the field. The total amount of pesticide applied when the threshold is decreased to $S_{t h}=3$ and increased to $S_{t h}=5$, compared with the original threshold $S_{t h}=4$ is shown in Table 6 . One obvious conclusion arising from the results in the table is that the amount of pesticide applied is reduced when a larger value of the threshold is used in the pesticide application protocol. It is interesting to note, that pesticide consumption decreases quite significantly when the threshold is increased. In the extreme case of the Stoney Lawn field on 18.02.2016 (line 4 of Table 6) we originally have the average trap count $S=9.13$ (cf. 4). Applying the threshold $S_{t h}=3$ requires $50 \%$ of the field area to be treated with pesticide and reduces the average trap count to $S_{\text {new }}=2.67$ while applying the threshold $S_{t h}=5$ requires half that area to be treated $(M=26.25 \%)$ and leaves the average trap count after pesticide application as $S_{\text {new }}=4.97$.

It is readily concluded from the above examples that targeted use of pesticide allows one to decrease significantly the amount of pesticide required to reduce the average trap count below a defined threshold. However, one problem with the protocol is that it is not clear from our use of the average trap count above whether it results in the optimal choice of traps for pesticide targeting. Consider, for example, a relatively low average trap count, such that just one trap has to be targeted to meet the condition $S \leq S_{t h}$. In cases such as in Fig. 4 f(i) there is one trap count that is considerably higher than the others and thus identifying a clear target for pesticide application. However, in Fig. 4e(i) there are several trap counts that are much higher than the rest, and targeting one trap in this case will reduce the average trap count to below the threshold while having little effect on the overall spatial distribution of slugs in the field (which is partly determined by environmental factors). Moreover, while the protocol reduces the average trap count below the threshold by making the average number of slugs in each selected patch lower, it does not result in smaller slug patches. In the case of the Stoney Lawn field, 14.01.2016, with threshold $S_{t h}=5$, targeted application of pesticide met the condition $S<S_{\text {th }}$ yet it resulted in 93 traps having been left untargeted out of 100 and patchy distribution of slugs has been preserved. Hence, the targeted use of pesticide resulting from the protocol based on the average trap count alone, neglects the patch size which is another important property of heterogeneous spatial slug distribution. In the next section we discuss possiblemodifications of the protocol that addresses explicitly the spatial distribution of slugs. 


\section{Multi-parametric identification of patches in the tar- geted use of the pesticide protocol}

It has been argued in the previous section that the threshold-based protocol does not take into account spatial characteristics of discontinuous (patchy) distributions and ittherefore remains uncertain whether the protocol offers an optimal selection of spatial sub-domains where pesticide should be applied. Uncertainty associated with the choice of traps for targeting when the spatial structure of slug distribution is neglected can be illustrated by the following simple example. Consider a linear transect across a hypothetical field where trap counts in traps spaced at equal distances are $[2,6,27,15,8,6,7,19,11,4]$. The average trap count is $S=10.4$. For the sake of simplicity in this example we assume that if a trap is targeted then pesticide will be applied over that trap only and neighbouring traps will not be caught in the area of pesticide application as can happen in the two-dimensional domains. Fig. 5 a shows the contribution of each trap count towards the average trap count (see stacked block $A$ ). In the pesticide application protocolbased on thesinglethreshold value $S_{t h}$ as explainedin Section 3.1 the traps are targeted one by one, starting with the largest trap count and descending towards the smallest, until the average trap count $S$ after pesticide has been applied is less than the threshold $S_{t h}$. However, the above method of selecting traps for targeting is not unique. It can be seen from the figure there are several alternative combinations of trap counts which collectively meet the condition $S<S_{t h}$ (see stacks $B, C$ and $D$ in Fig. 5a) and it remains unclear from the protocol which combination is optimal.

Our algorithm for trap selection in Section 3.1 is based on the implicit definition of slug patch as a sub-domain with the high slug density. This definition does not take into account the spatial size of any single patch as the slug abundance is considered as the dominant feature of patch. An alternative definition, however, could be based on the geometric size of patch where the area occupied by the patch is considered as its main characteristic and the following protocol takes this factor into account.

\subsection{Definition of patch characteristics: slug abundance in patch vs. patch size}

As we could see in Section 2 the most straightforward way to define a spatial patch would be to label any isolated spatial sub-domain with the non-zero population density as a patch. The above definition accounts for patch size automatically, yet it may result in redundant pesticide application when the population density across the entire field is low. We therefore proceed with a more prescribed definition of patch size where only patches with relatively high population abundance will be considered.

The concept underpinning the modified definition of patch size is to identify key trap counts to be targeted, and then by examining the neighbouring trap catches, determine whether the patch has ended or whether it also covers the area assessed by these traps. The above suggestion requires two thresholds $S_{u}$ and $S_{l}$ (i.e. the upper and lower thresholds). The 

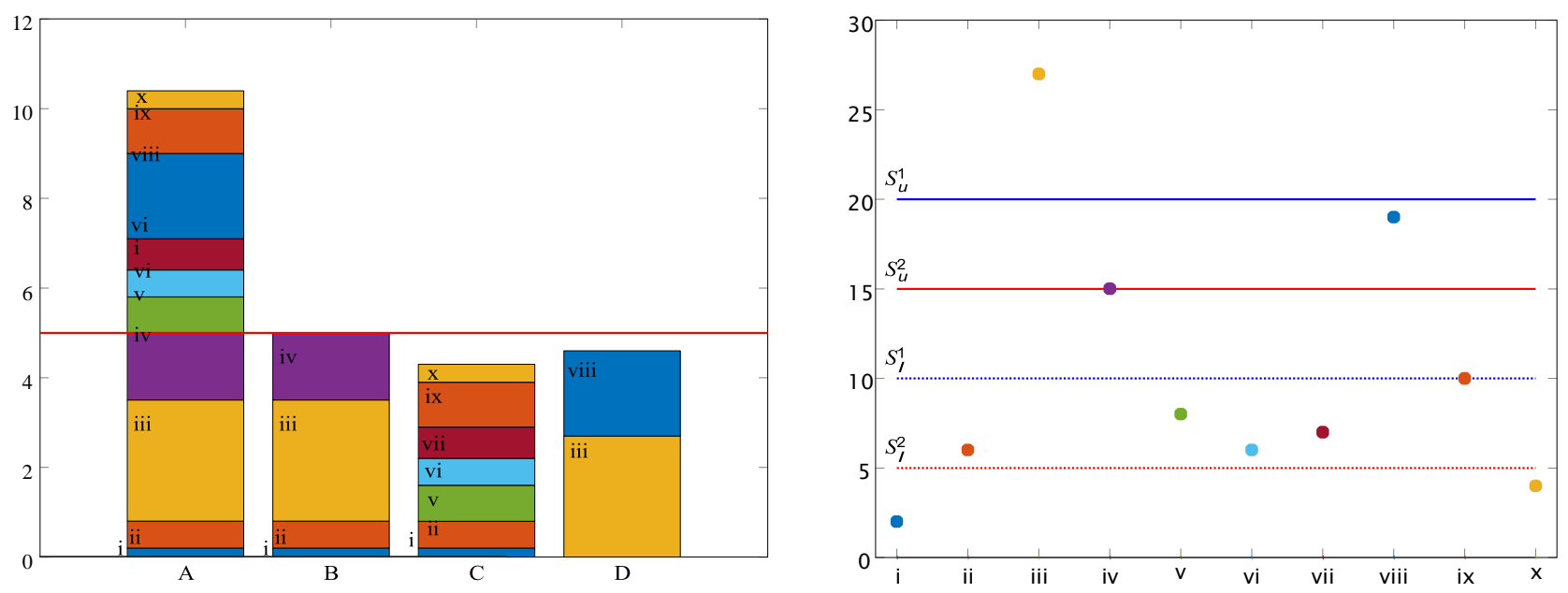

Figure 5: A transect test case of trap counts. Roman numerals $\mathrm{i}-\mathrm{x}$ are used for traps numbering. (a) The threshold based approach (see Section 3). Each coloured block in stacked bar A represents the contribution of each trap towards the average $S=10.4$ (going from trap i at the bottom to trap x at the top). The threshold is set at $S_{t h}=5$; see red solid horizontal line in the figure. To satisfy our aim of reducing the average as $S \leq S_{t h}$, we need to remove blocks until the trap counts stack is smaller than the threshold. That can be achieved in various ways; see stacks $B, C$ and $D$ in the figure. (b) The 'double threshold' approach. The trap count in each trap along the transect is shown as a coloured stem in the figure. The solid lines are the upper threshold $S_{u}$ set to 20 (blue) and 15 (red). The dashed lines are the lower threshold $S_{l}$ set to 10 (blue) and 5 (red).

procedure to find patches is then to firstly identify all trap counts with a value greater than or equal to upper threshold $S_{u}$ and mark those traps in the sampling grid. For each ofthese points in the grid, we then consider the neighbouring trap counts. If a neighbouring trap count has a value greater than or equal to the lower threshold $S_{l}$, then it is included as part of the patch. The process is then repeated iteratively for all new traps included in the patch until no neighbouring trap catch is found to exceed $S_{l}$.

Consider again the transect example introduced in Fig. 5 and let us now show the trap count in each trap along the transect as a coloured stem in Fig. 5b where trap numeration and colour scheme is the same as in Fig. 5a. Let us establish upper threshold $S_{l}^{1}=20$ (blue solid horizontal line) and lower threshold $S^{1}=10$ (blue dotted horizontal line). Then there is only the trap count in trap iii which is greater than the upper threshold and there willbe only one small patch around trap iii and trap iv, as all neighbouring trap counts are below the lower threshold. However if we decrease the lower threshold and use $\underset{u}{S^{1}=20}$ and $S^{2}=5$, shown with the solid blue and red dotted line, then there will be one large patch covering the area around trap ii to trap ix, as all of these traps have trap counts exceeding the lower threshold and at least one of the trap counts is above the upper threshold. If we also decrease the upper threshold and use $S_{u}^{2}=15$ and $S_{l}^{2}=5$ (the two red horizontal lines in Fig. 5b), then 


\begin{tabular}{|l|l|l|l|l|}
\hline$S_{u}$ & $P$ & $T \%$ & $S_{\text {new }}$ & $M \%$ \\
\hline 5 & 9 & 38 & 0.43 & 73.75 \\
10 & 4 & 33 & 0.64 & 70.00 \\
20 & 3 & 30 & 0.97 & 67.50 \\
30 & 2 & 26 & 1.56 & 57.50 \\
40 & 1 & 22 & 2.29 & 50 \\
\hline
\end{tabular}

Table 7: A comparison of the results of applying the double threshold protocol using data from the Stoney Lawn field on 14.01.2016, but with different upper threshold values $S_{u}$. The lower threshold is $S_{l}=5$. P is the number of patches, $T$ is the percentage of traps targeted for pesticide application, $S_{\text {new }}$ and $M \%$ are as in Table 4.

we also get one large patch covering trap ii to trap ix as lowering the upper threshold does not result in marking any new traps outside of this area. Finally, if we consider $S_{u}^{2}=15$ and $S_{l}^{1}=10$, there are two trap counts in Fig. $5 \mathrm{~b}$ that are higher than the upper threshold, two trap counts higher than the lower threshold and there are several traps between them with the trap counts below the lower threshold. Therefore we will have two distinct patches, one covering traps iii and iv and one covering traps viii and ix.

\subsection{Example of the two-parametric patch identification}

Let us label the pesticide procedure where the lower and upper thresholds are introduced as a 'double threshold' protocol. We conclude from the above consideration that the upper threshold $S_{u}$ largely determines the number of patches to be targeted and the lower threshold $S_{l}$ determines both the size of those patches and the number of situations where two patches can be considered as having merged into one. Let us further illustrate the 'double threshold' protocol by applying it to the Stoney Lawn field on 14.01.2016 (see data in Table 3 and the spatial distribution in Fig. 2). This protocol yields different results to the 'single threshold' based approach discussed in Section 3.

a

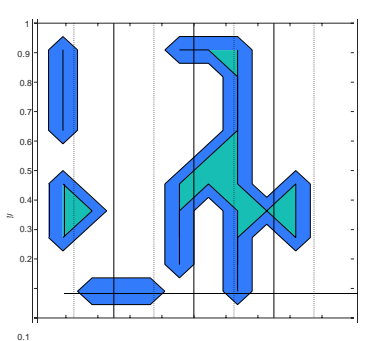

$\mathrm{b}$

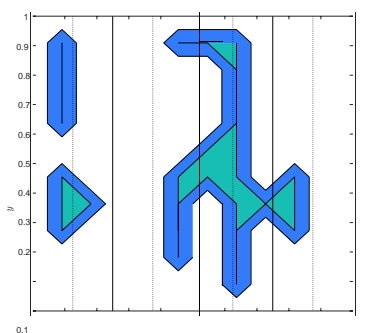

c

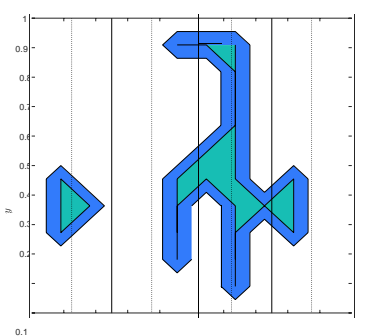

d

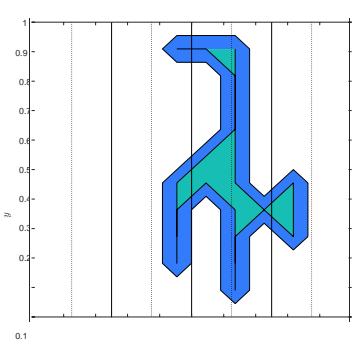

Figure 6: A comparison between the results of the patch definition at the Stoney Lawn field on 14.01.2016 with the lower threshold set to $S_{l}=5$ and varying upper thresholds. Contour plots 
showing slug patches (areas of light green colour in the figure) with boundary region (blue colour). (a) Upper threshold is $S_{u}=10$ (b) $S_{u}=20$ (c) $S_{u}=30$ (d) $S_{u}=40$. 


\begin{tabular}{|l|l|l|l|l|}
\hline$S_{l}$ & $P$ & $T \%$ & $S_{\text {new }}$ & $M \%$ \\
\hline 20 & 4 & 6 & 3.95 & 15.0 \\
15 & 4 & 10 & 3.06 & 25.00 \\
10 & 3 & 16 & 1.96 & 43.75 \\
6 & 3 & 24 & 0.97 & 67.50 \\
4 & 3 & 41 & 0.23 & 83.75 \\
3 & 2 & 54 & 0.0 & 100 \\
2 & 2 & 71 & 0.0 & 100 \\
\hline
\end{tabular}

Table 8: A comparison of the results of applying the double threshold protocol using different lower thresholds to data from the Stoney Lawn field on 14.01.16. The upper threshold is $S_{u}$ $=20$. The legend is the same as in Table 7 .

Table 7 shows the results of the 'double threshold' protocol when we fix the lower threshold as $S_{l}=5$ and vary the upper threshold from $S_{u}=5$ to $S_{u}=40$. When the upper threshold is increased, we can see that the number of patches and targeted traps decreases as expected. The results of this can be seen in Fig. 6 where $S_{u}$ is set to 10, 20,30 and 40. In the figure, the size of the individual patches is always the same but the number of patches decreases as $S_{u}$ increases. When $S_{u}=10$ there will be at least one trap in each of the four patches that has a trap count higher than $S_{u}$; see Fig. 6a. However only one of those patches has a trap with a count exceeding $S_{u}=40$; see Fig. $6 \mathrm{~d}$.

Consider now Table 8 where we fix the upper threshold to $S_{u}=20$ and vary the lower threshold $S_{l}$ from $S_{l}=2$ to $S_{l}=20$. This allows us to confirm how the two thresholds define the patches and how varying them changes outcomes.

As explained above, the upper threshold controls the number of patches and the lower threshold determines the size of the individual patch. When we decrease the lower threshold, we can see that the number of targeted traps always increases but the number of patches will either decrease or stay the same. This is because the maximum number of patches with a fixed $S_{u}$ will be found when $S_{l}=S_{u}$ and the traps that have counts that exceed this threshold

a

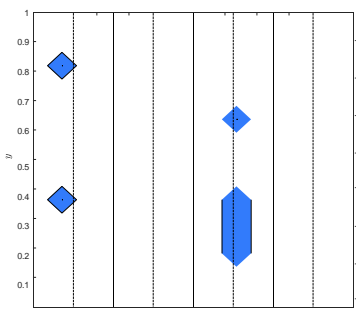

$\mathrm{b}$

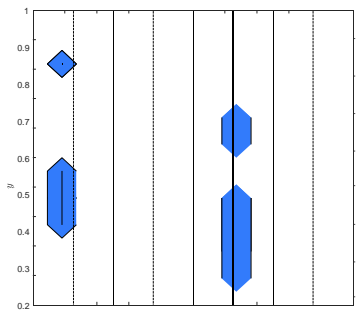

$\mathrm{c}$

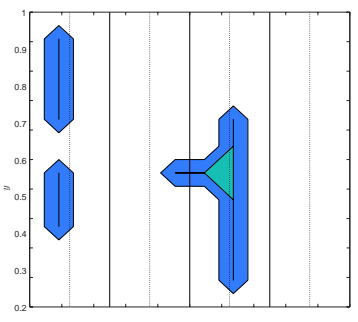

d

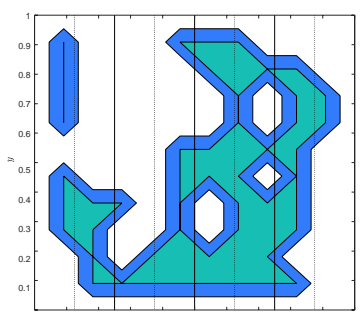

Figure 7: A comparison between the results of the patch definition at the Stoney Lawn field on 14.01.2016 with the upper threshold $\boldsymbol{S}_{u}=20$ set to 20 and varying lower thresholds. Contour 
plot legend as in Fig. 6.(a) $S_{l}=20(b) S_{l}=15(c) S_{l}=10(d) S_{l}=3$. 
often belong to separate patches. This can be seen in Fig. 7(a) and in the top row of table 8. There are six traps targeted when $S_{u}=S_{l}=20$ but three of them are in the same patch. As $S_{l}$ decreases, more traps that were neighbouring those in the patch will be incorporated into that patch as soon as their trap count exceeds the new value of $S_{l}$; see Fig. 7(b)-(c). Eventually, with decreasing $S_{l}$, the patches will become large enough to merge together and hence we have a decreasing number of patches; see Fig. 7(d).

The 'double threshold' approach offers a stricter control on the patch size in comparison with the 'single threshold'-based protocol. Meanwhileapplying the double threshold protocol on its own does not guarantee that the average trap count will be $S<S_{\text {th }}$ after pesticide application. Hence our next goal is to combine both 'single threshold' and 'double threshold' approaches into one protocol, therefore ensuring that a new average trap count is lower than the threshold $S_{t h}$ while also acknowledging the spatial properties of the patches. The details of a unified protocol are explained below.

\subsection{A unified approach to definition of slug patch}

A unified approach summarises our discussion about an optimal definition of slug patch in a targeted use of pesticide procedure where we now design a pesticide application protocol to take into account both the slug density in the patch and the patch size. The protocol utilizes the algorithms discussed in Sections 3.1 and 4.1 and consists of two respective steps. The first step is, as in the single threshold based approach developed in 3.1, to determine the traps that must be targeted to reduce the average trap count below the given threshold. That should reveal slug patches with the high slug density irrespective of their spatial size. Wethen follow the procedure from the 'double threshold' approach developed in Section 4.1 and apply lower threshold $S_{l}$ to the traps identified for targeting in step 1. Therefore, any neighbouring traps with a trap count about $S_{l}$ will also be targeted thus defining the size of the patches requiring treatment. For simplicity, it may be appropriate to set $S_{l}=S_{t h}$, i.e. all neighbouring traps with counts higher or equal to the threshold should also be targeted.

\begin{tabular}{|l|l|l|l|l|}
\hline$S_{t h}$ & $S^{1}$ & $M^{1} \%$ & $S^{2}$ & $M^{2} \%$ \\
\hline 3 & 2.91 & 26.2 & 0 & 100 \\
4 & 3.95 & 15.0 & 0.23 & 83.75 \\
5 & 4.71 & 7.50 & 1.56 & 57.50 \\
6 & 5.49 & 2.50 & 2.29 & 50.00 \\
\hline
\end{tabular}

Table 9: Results from the combined protocol on Stoney Lawn 14.01.16 with the varying threshold $S_{\text {th. }}$ The field had an average trap count of $S=6.03$ prior to pesticide application. The lower threshold at the second step of the combined protocol is $S_{l}=S_{t h} . S^{1}$ and $M^{1} \%$ are the average trap count and the area treated with pesticide that would result from treatment decisions being made at the first step of the combined protocol (see details in the text), $S^{2}$ and $M^{2} \%$ are the average trap count after pesticide application and the area treated with pesticide following decisions made at the second step of the protocol. 
a

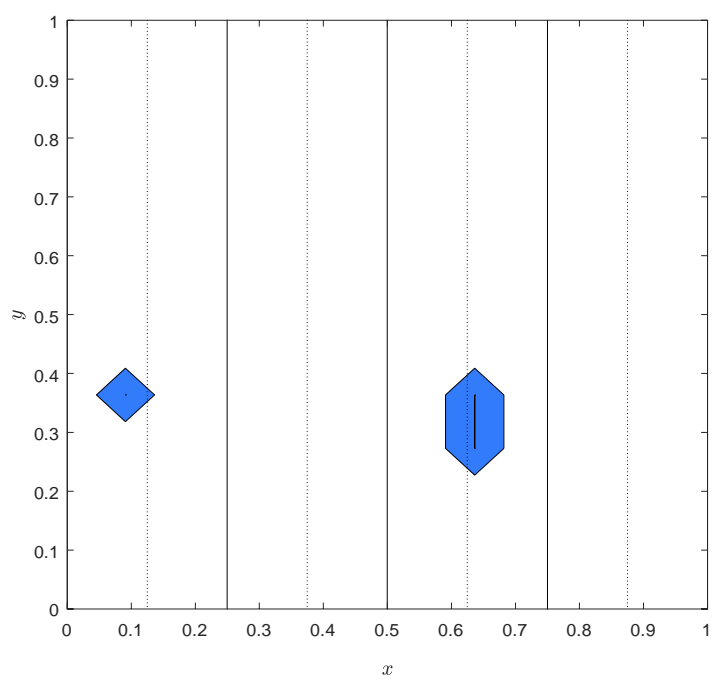

$\mathrm{b}$

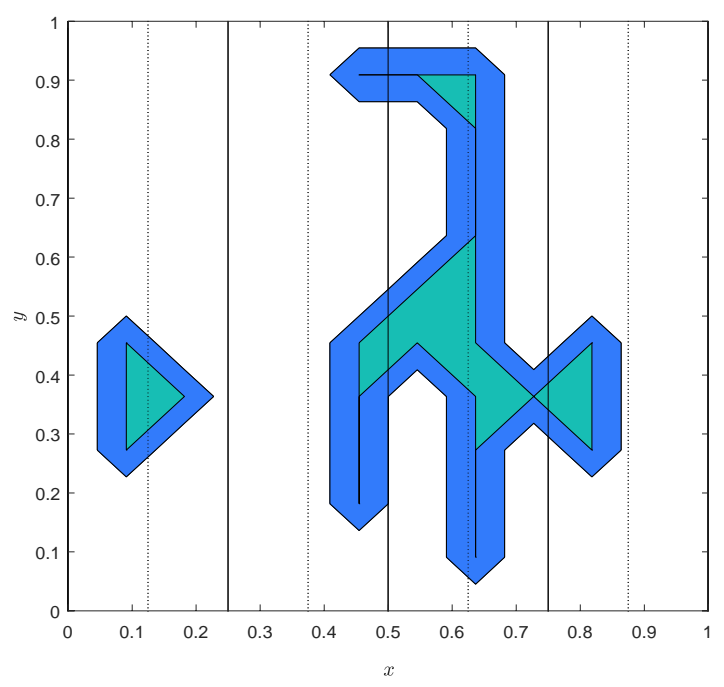

Figure 8: The targeted trap counts in the Stoney Lawn field on 14.01.16. Contour plot legend as in Fig. 6. (a) the single threshold approach applied only $\left(S_{t h}=5\right)$ and $(b)$ an additional lower threshold applied $\left(S_{l}=5\right)$.

Consider our baseline example of the Stoney Lawn field on 14.01.16. In this case $T=3$ traps are targeted in two patches when we set the threshold $S_{t h}=5$. If a pesticide was applied this would reduce the average trap count from $S=6.03$ to $S=4.71$ and it would require $M$ $=7.5 \%$ of the pesticide amount used to treat the whole field (cf. data in Table 6). However, if instead of applying pesticide we go to step 2 of our unified protocol, then additional traps are targeted under the condition that trap count $C_{i}$ in the $i$ th trap is $C_{i}>S_{l}$ provided the $i$ th trap is a neighbour of any of the three traps initially marked for targeting by step 1 . If we then apply the lower threshold $S_{l}=S_{t h}=5$, this increases the number of traps targeted to $T$ $=26$, still making up two individual patches as shown in Fig. 8, and the new average trap count is $S_{\text {new }}=1.56$ after pesticide application. The area treated with pesticide relates to the amount used and it increases from $M=7.5 \%$ to $M=57.5 \%$ mass units.

Table 9 shows the results of the combined protocol after the first and second step of patch identification when the threshold $S_{t h}$ is varied. In the table, $S^{1}$ and $M^{1} \%$ are the average trap count and the area treated with pesticide if decision to treat is made at the first step of the combined protocol. Those values are compared to $S^{2}$ and $M^{2} \%$ which are the average trap count after pesticide application and the area treated when decision making occurs at step 2. It is seen from the table that the addition of a lower threshold that takes into account patch size significantly reduces the average trap count $S_{\text {new }}$ after pesticide application yet it increases the amount of pesticide used. When $S_{t h}=S_{l}=6$, after applying the combined approach the average trap count is reduced to $S=2.29$, far less than the threshold $S_{t h}$ but it requires $M=50 \%$ of the field to be treated. In contrast, the single threshold based protocol (i.e. the first step of the combined approach) would use only $M=26.2 \%$ of the total pesticide to reduce the average trap count to $S=2.91$. 
It can be concluded from the study caseconsidered in thissection that the combined protocol uses pesticide in a less efficient way in comparison to the standard threshold based approach. Nevertheless, the combined protocol maybe more effectivein real world applications as it may be important to target large areas to slow down or stop slug patches reforming as may happen if smaller areas are targeted and the surrounding areas have reasonably large trap counts.

\section{Conclusions}

In the present paper we have established a theoretical basis for a prototype targeted pesticide application protocol that allows the selective control of a pest population in agricultural fields. The protocol is based on the analysis of a strongly heterogeneous spatial distribution of the pest population using field data collected from commercial fields. Although the conclusions are applicable to many pest species, the grey field slug (D. reticulatum) was selected as the baseline case for our study as there is extensive evidence that the spatial distribution this species in arable crops is strongly heterogeneous South (1992); Bohan et al. (2000); Archard et al. (2004); Mueller-Warrant et al. (2014). Our approach identifies the areas of high slug density (slug patches) and pesticide is applied selectively to those areas alone, differing from many existing commercial protocols in which the entire field is uniformly treated. Our model demonstrates that the approach results in considerable savings in the total crop area treated, and thus the amount of pesticide used, potentially contributing to current environmental sustainability priorities and yielding a small positive effect on profit margins.

While the suggested protocol provides a platform from which multiple opportunities relating to selective application of pesticides can be pursued, it requires further validation and development to facilitate cost-efficacy and commercial viability before it can be considered for use by practitioners. The most challenging issue that has still to be resolved is definition of a spatial patch. We have argued in Section 3 that a generic definition of a patch as any spatial subdomain with a closed boundary that has a non-zero population density is not efficient when targeted use of pesticide is considered. Indeed, the above definition implies that pesticide is applied to every patch in the field, irrespective of its size or the pest density within it. Under more realistic conditions, the definition of patch will require some additional constraints. Depending on economic and environmental goals, practitioners will require the identification of sufficiently large patches with population density that exceeds defined level. It has been shown in Section 4 that different approaches to defining a patch will lead to different conclusions about the spatial pattern of pest distribution and consequential variability in the area of a crop identified for treatment with pesticides (cf. Fig. 6 and Fig. 7). We therefore conclude that the definition of a spatial patch has to be carefully considered before it can be employed in a targeted-treatment protocol. Heterogeneous spatial distributions of various animals have been a focus of intense study in past decades and reliable statistical measures have been developed to classify the degree of animal aggregation in a spatial domain (e.g. see Taylor (1984); Waters (1959)). However, the extent to which it is possible to exploit previous research results in spatial analysis undertaken as a part of an investigation of the problem of targeted 
use of pesticides is unclear. The definition of a spatial patch requires careful investigation in future work as it becomes dependent on the conditions of the control protocol. Furthermore, the targeted use of pesticide procedure discussed in the paper requires 'temporal stability' of patches. Since the decision about patch boundaries is essentially based on the analysis of trap counts, the time scale of the trapping protocol implies that the procedure cannot be applied to species who form volatile patches where the patch boundaries change rapidly with time. The definition of temporal stability of a patch depends on the definition of patch per se and those two issues should be investigated together.

Another important question that our study raises is the economic reliability of threshold values. Established monitoring and control protocols are based on the assumption that treating pest populations to prevent them reaching or exceeding defined threshold levels should avoid (or at least alleviate) economic damage being caused by the pest. The targeted use of pesticide procedure relies heavily on the above assumption, but the present paper demonstrates that it can result in significant numbers of pests (in our case slugs) remaining in locations surrounding those that have been targeted. Thus, despite having reduced the population to sub-threshold level, it is possible that the remaining individuals may form a nucleus for pest resurgence after the efficacy of the pesticide has declined, resulting in rapid reformation of patches, particularly if there is an environmental driver for clustering in those areas. It might be argued that the current practical use of thresholds have been based partly on the assumption that a large proportion of the population is removed after treatment, and thus the economic calculations underpinning them incorporate these considerations (firstly by reducing post-treatment crop losses sufficiently to make treatment cost-effective and secondly by reducing the rate at which resurgence occurs). On the other hand, approaches in which treatments are applied to entire fields may result in the post-application average trap count being unnecessarily much smaller than the threshold value. The current study offers a basis from which recalculation of thresholds can contribute to more sustainable use by application of reduced volumes of pesticide through more careful targeting. It has been demonstrated in the paper that definition of more accurate thresholds will require more information about heterogeneous spatial distributions of pest populations and two control parameters quantifying both the pest abundance in each patch and the patch size will be required in monitoring and control protocols.

Finally, a challenging question closely related to the use of thresholds, is the issue of accurate evaluation of the pest abundance. In the present study we have assumed that the average trap count is a reliable estimate of the pest population in the field because a very fine sampling grid has been used in our study. While average trap counts have long been used to provide information on population abundance in ecological applications or when making pest management decisions Anderson et al. (2013); Disney (1986); Walters et al. (2003), it has been recently demonstrated in our work Petrovskaya and Embleton (2013); Petrovskaya (2018) that the spatial heterogeneity (patches) presenting in a spatial pattern of the population can make the problem of population abundance evaluation challenging. The problem is exacerbated by the fact that a coarse sampling grid is usually employed in a monitoring/control protocol and it has been shown Petrovskaya et al. (2018) that in the latter case the estimate of population abundance becomes essentially a random variable. Further investigation of this 
issue is required.

\section{Acknowledgments}

This study was partially supported by AHDB through Project 214-0009118 'Utilising the patchy distribution of slugs to optimise targeting of control: improved sustainability through precision application.' J.R.E. acknowledges support in the form of a Research Associateship from the School of Mathematics, University of Birmingham.

\section{References}

M.C.R.Alavanja, M.K.Ross, M.R.Bonner. (2013) Increased cancer burden among pesticide applicators and others due to pesticide exposure. CA: A Cancer Journal for Clinicians 63:120142

A. Alyokhin, M. Baker, D. Mota-Sanchez et al. (2008) Colorado potato beetle resistance to insecticides. American Journal of Potato Research 85:395-413.

J.T. Anderson, F.L.Zilli, L.Montalto, M.R. Marchese, M.McKinney, Y.L.Park. (2013) Sampling and processing aquatic and terrestrial invertebrates in wetlands. In; J.Anderson, C.Davis, Eds.; Wetland Techniques; Springer: Dordrecht, The Netherlands.

G.Archard, D. Bohan, L.Hughes, C.Wiltshire. (2004) Spatial sampling to detect slug abundance in an arable field. Annals of Applied Biology 145:165-173.

T. C. Bailey, A. C. Gatrell. (1995) Interactive Spatial Data Analysis. Pearson Education Ltd.

D. Bohan, D. Glen, C. Wiltshire, L. Hughes. (2000) Parametric intensity and spatial arrangement of the terrestrial mollusc herbivores Deroceras reticulatum and Arion intermedius. Journal of Animal Ecology 69:1031-1046.

R. J. Brenner, D. A. Focks, R. T. Arbogast, D. K. Weaver, and D. Shuman. (1998) Practi- cal use of spatial analysis in precision targeting for integrated pest management. American Entomologist, 44(2):79-102.

D. L.Brown, D. K. Giles, M. N. Oliver and P. Klassen. (2008) Targeted spray technology to reduce pesticide in runoff from dormant orchards. Crop Protection, 27(3-5):545-552.

P.M.Davis. (1994) Statistics for describing populations. In: Pedigo L. P., Buntin G. D. (eds) Handbook of sampling methods for arthropods in agriculture CRC Press, Boca Raton, USA $33-54$

DEFRA. 2016. Drinking Water 2015; A summary of the Chief Inspectorate's report for drinking water in England. London: Drinking Water Inspectorate. 
H.R.Disney. (1986) Assessments using invertebrates: Posing the problem. In: Usher, M.B., Ed.; Wildlife Conservation Evaluation; Chapman and Hall: London, UK, 271-293.

S. Fleischer, P. Blom, and R. Weisz. (1999) Sampling in precision IPM: when the objective is a map. Phytopathology, 89(11):1112-1118.

E. Forbes, M. Back, A. Brooks, N.B. Petrovskaya, S.V. Petrovskii, T. Pope, and K. F. A. Walters. (2017) Sustainable management of slugs in commercial fields: assessing the potential for targeting control measures. in: Aspects of Applied Biology 134, Association of Applied Biologists, 89-96.

E. J. Gustafson. (1998) Quantifying landscape spatial pattern: what is the state of the art? Ecosystems, 1(2):143-156.

P.C.Jepson, J.R.M.Thacker.(1990) Analysis of the spatial component of pesticide side-effects on non-target invertebrate populations and its relevance to hazard analysis. Functional Ecol- ogy $4: 349-355$

MATLAB (2017): https://uk.mathworks.com/help/images/index.html

G. A, Matthews (2014) Pesticide Application Methods 4th edition. Wiley Blackwell, Oxford. 517 pp.

G. A. Matthews (2016) Pesticides Health Safety and the Environment. 2nd edition. Wiley Blackwell, Oxford 277 pp.

P. C. Miller. (2003) Patch spraying: future role of electronics in limiting pesticide use. Pest Management Science, 59(5):566-574.

G. Mueller-Warrant, N. Anderson, C. Sullivan, G. Whittaker. K. Trippe, (2014) Can knowledge of spatial variability in slug populations help improve stand establishment? Seed Production Research, Oregon State University 151: 4-13

J.D. Nichols (2014) The role of abundance estimates in conservation decision-making. In: Verdade L., Lyra-Jorge M., Pica C. (eds) Applied Ecology and Human Dimensions in Biological Conservation. Springer, Berlin, Heidelberg, doi.org/10.1007/978-3-642-54751-5-8

N.B.Petrovskaya, N.L.Embleton. (2013) Evaluation of peak functions on ultra-coarse grids. Proc. R. Soc. A, 469, 20120665, doi:10.1098/rspa.2012.0665.

N.B.Petrovskaya. (2018) 'Catch me if you can': evaluating the population size in the presence of a spatial pattern. Ecol. Complex., 34:100-110.

N.B. Petrovskaya, E. Forbes, S.V. Petrovskii, K.F.A. Walters. (2018) Towards the develop- ment of a more accurate monitoring procedure for invertebrate populations, in the pres- ence of unknown spatial pattern of population distribution in the field. Insects, 9(1), 29; doi:10.3390/insects9010029 
D.Pimentel(ed). (1997)Techniques for reducing pesticide use: economic and environmental benefits. John Wiley \& Sons, New York

D.Pimentel, A.Greiner. (1997) Environmental and socio-economic costs of pesticide use. In: Pimentel D (ed) Techniques for reducing pesticide use: economic and environmental benefits. John Wiley \& Sons, New York p 51-78

D. Pimentel. (2009) Pesticides and pest control. In: Peshin R, Dhawan AK (eds) Integrated pest management: innovation-development process. Springer, Berlin 1:83-87

C. Port, G. Port. (1986) The biology and behaviour of slugs in relation to crop damage and control. Agricultural Zoology Reviews, 1:255-299.

P. J. Sammons, T. Furukawa, and A. Bulgin. (2005) Autonomous pesticide spraying robot for use in a greenhouse. In: Australian Conference on Robotics and Automation, 1-9.

G.W.Snedecor, W.G.Cochran. (1980) Statistical Methods. The Iowa State Iniversity Press, Ames.

F.Sohrabi, P.Shishehbor, M.Saber, M.S.Mosaddegh. (2013) Lethal and sublethal effects of imidacloprid and buprofezin on the sweetpotato whitefly parasitoid Eretmocerus mundus (Hymenoptera: Aphelinidae). Crop Protection 45:98-103

N.W.Sotherton, P.A.Robertson, and S.D.Dowell. (1993) Manipulating pesticide use to increase the production of wild game birds in Britain. National Quail Symposium Proceedings: Vol. 3, Article 13. In: K. E. Church and T. V. Dailey (eds). Quail III: national quail symposium. Kansas Dep. Wildl. and Parks, Pratt, 92-101.

A. South, (1992) Terrestrial Slugs: Biology, Ecology and Control. London: Chapman \& Hall.

V.M.Stern, R.F.Smith, R.van den Bosch, K.S.Hagen. (1959) The integration of chemical and biological control of the spotted alfalfa aphid. Part I. The integrated control concept. Hilgardia 29:81-101

L. R. Taylor (1984) Assessing and interpreting the spatial distributions of insect populations. Ann. Rev. Entomol., 29:321-57

S. Twining, J. Clarke, S. Cook, S. Ellis, P. Gladders, F. Ritchie, S. Wynn. (2009) Pesticide availability for potatoes following revision of Directive 91/414/EEC: Impact assessments and identification of research priorities. Project report 2009/2. Warwickshire, UK: Potato Council, AHDB.

K.F.A. Walters, J.E.B. Young, B. Kromp, P.D.Cox. (2003) Management of oilseed rape pests. In: D.V.Alford, Ed.; BiologicalControl of Pests of Oilseed Rape; BlackwellScience: Oxford, UK, 43-71.

W.E. Waters. (1959) A Quantitative measure of aggregation in insects. J. Economic Entomology, 52(6):1180-1184. 


\section{Appendix A: GPS coordinate transformation}

In this section we explain brieflya process that willtransform coordinatesfrom the unitsquare, as we have used in Section 2, to actual GPS coordinates in the field. The coordinates of the four corners traps in the sampling grid are required as an input and we then geometrically transform those coordinates into the unit square to be further used in the MATLAB software. We can then use the transformation to convert any GPS coordinates from the field into unit square coordinates. The unit square can then be transformed back to the GPS coordinates and any coordinate from the unit square can be converted to GPS coordinates. This will be necessary when determining the start and finish points for applying pesticide in the field.

We consider the GPS coordinates $(x, y)$ and the four corner traps to be $\left(x_{i}, y_{i}\right)$ for $i=1, \ldots, 4$ going clockwise starting from the bottom left point as shown in Fig. 9. The coordinates $(x, y)$ are then changed according to the transformation that turns the corner coordinates into a unit square. However, we need the traps to be in the interior of the unit square and not on the boundary, therefore we position the corner points at distances of $\frac{h}{2}$ from the boundary:

$\left(\frac{h}{2}, \frac{h}{2}\right),\left(\frac{h}{2} 1-\frac{h}{2}\right),\left(1-\frac{h}{2}, \frac{1}{2}-\frac{h}{2}\right)$ and $\left(1-\frac{h}{h}, \frac{h}{2}\right.$ where $h$ is the distance between traps. The first step is to translate the coordinates so that $\left(x_{1}, y_{1}\right)=(0,0)$ so that we can make our rotation and scaling transformation. Therefore

$$
\left(x_{t}, y_{t}\right)=\left(x-x_{1}, y-y_{1}\right)
$$

As we know the traps are already set out in a square of 10om, the only remaining transformations required are rotation and rescaling. The rotation matrix is given as

$$
M_{r}=\begin{array}{cc}
\cos (\theta) & -\sin (\theta)^{r} \\
\sin (\theta) & \cos (\theta)
\end{array}
$$
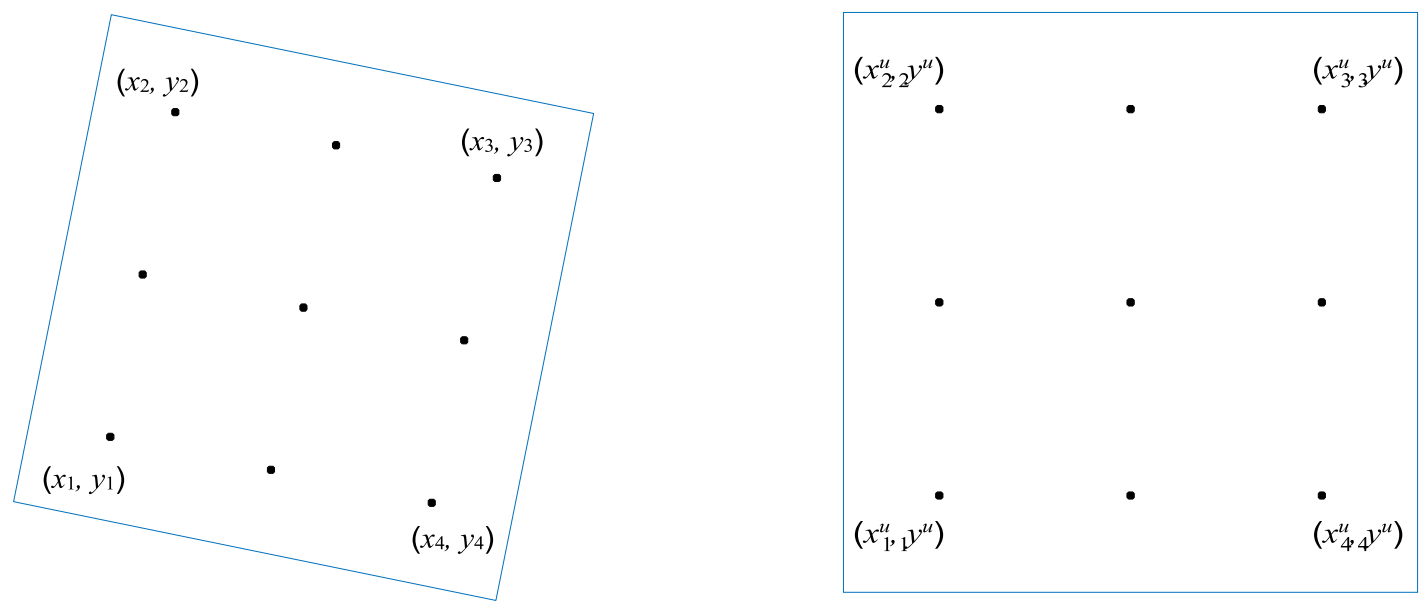

Figure 9: An example of a field with nine traps and the coordinates of the four corner traps transformed into the unit square. 
and the scaling matrix is

$$
M_{s}=\begin{array}{cc}
\frac{1-h}{x} \underline{h} & 0 \\
0 & \frac{1-h}{y} \underline{h}
\end{array},
$$

where

$$
\theta=\tan ^{1} \quad \frac{y_{4}-y_{1}}{x_{4}-x_{1}}
$$

is the angle from the horizontal between the bottom left and right corners and

$$
\begin{aligned}
& x_{l}=\sqrt{\left(x_{1}-x_{4}\right)^{2}+\left(y_{1}-y_{4}\right)^{2}}, \\
& y_{l}=\sqrt{\left(x_{1}-x_{2}\right)^{2}+\left(y_{1}-y_{2}\right)^{2}}
\end{aligned}
$$

are the distances between $\left(x_{1}, y_{1}\right)$ and $\left(x_{4}, y_{4}\right)$, and $\left(x_{1}, y_{1}\right)$ and $\left(x_{2}, y_{2}\right)$ which reduces the size from the distance between the corners to a distance of $1-h$. Although in this report we only take the case of a square grid of traps when measured in metres, this is not necessarily square when taking GPS coordinates, therefore the scalings in the $x$ and $y$ directions are not the same.

By multiplying these matrices together we are left with one transformation matrix to apply to the translated $\left(x_{t}, y_{t}\right)$ to get our new coordinates for the unit square $\left(x_{u}, y_{u}\right)$.

$$
\begin{aligned}
& \begin{array}{lllll}
\cos (\theta) & -\sin (\theta)^{r} & \underline{1-h} & 0 & \underline{(1-h) \cos (\theta)}
\end{array}
\end{aligned}
$$

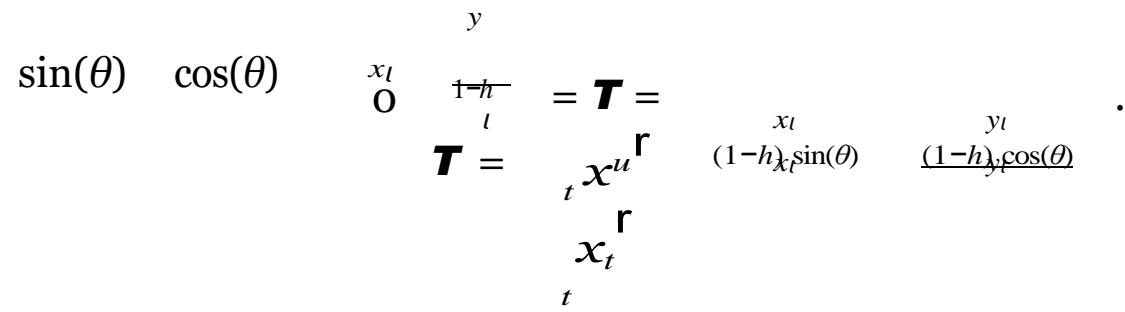

$$
\begin{aligned}
& y_{u} \quad y_{t}
\end{aligned}
$$

Then we reposition away from the boundary so that

$$
\left(x^{u}, y^{u}\right)=\left(x^{u}+\frac{h}{-}, y^{u} h .\right.
$$

To find $(x, y)$ from coordinates in the unit square $\left(x^{u}, y^{u}\right)$, we translate the coordinates back to the unit square and use the inverse matrix to translate back to GPS coordinates.

where

$$
\begin{aligned}
& \left(x_{t}^{u}, y_{t}^{u}\right)=\left(x^{u}-\frac{h}{2}, y^{u}-\frac{h}{2}\right) . \\
& r_{y^{\prime}}^{x^{\prime}}=\boldsymbol{T} \underset{y_{t}^{u}}{\stackrel{r}{x}},
\end{aligned}
$$

$$
\boldsymbol{T}^{i}=\frac{(1-h) \cos (\theta)}{x_{l}} \frac{-(1-h) \sin (\theta)^{\prime}}{y_{l}}=\frac{1}{1} x_{l} \cos (\theta) \quad x_{l} \sin (\theta)^{r} .
$$




$$
\frac{(1-h) \sin (\theta)}{x_{l}} \quad \frac{(1-h) \cos (\theta)}{y_{l}} \quad(1-h)^{2}-y_{l} \sin (\theta) y_{l} \cos (\theta)
$$

Finally we have

$$
(x, y)=\left(x_{t}+x_{1}, y_{t}+y_{1}\right)
$$




\section{Appendix B: algorithm for threshold based protocol}

In this section we provide justification of our algorithm for marking traps for pesticide application discussed in Section 3. We analyse the average trap count by rewriting expression (1) in Section 3 as follows:

$$
S=\frac{1}{N}{ }_{i=1}^{\mathbf{N}} C_{i}=\frac{1}{N} \underset{i=1}{\mathbf{N}}\left(S_{t h}+C_{i}-S_{t h}\right)=S_{t h}+\frac{1}{N} \underset{i=1}{\mathbf{N}}\left(C_{i}-S_{t h}\right),
$$

where we notice that the difference $C_{i}-S_{t h}$ for any trap count $C_{i}$ can be negative, positive or zero value. Let $N_{1}$ traps have the trap count $C_{j}<S_{t h}, j=1,2, \cdots, N_{1}$, and $N_{2}$ traps have the trap count $C_{i} \geq S_{t h}, i=1,2, \cdots, N_{2}$, where $N_{1}+N_{2}=N$. We then rearrange the sum in (15) as

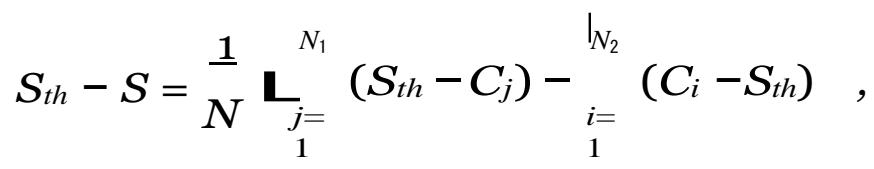

where both sums in (16) are positive because of the definition of trap counts $C_{j}$ and $C_{i}$.

Clearly the condition $S_{t h}-S>$ o holds if we have

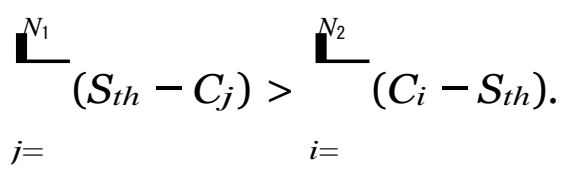

Let us find the highest trap count in the sum ${ }_{i=1}^{\mathbf{N}_{2}}\left(C_{i}-S_{t h}\right)$ and denote it as $C_{i^{*}}$. Suppose that the pesticide has been applied to the area around the trap $i_{*}$ only and all slugs have been killed in that area as a result of pesticide application. We can therefore consider the trap count in that trap as $C_{i^{*}}=0$ after pesticide application and the expression (16) becomes

$$
S_{t h}-S=\frac{1}{N} \bigsqcup_{j=1}^{N_{1}+1}\left(S_{t h}-C_{j}\right)-{ }_{i=1}^{N_{\mathbf{L}}-1}\left(C_{i}-S_{t h}\right),
$$

where the trap count $C_{i^{*}}=$ o now contributes to the first sum on the right-hand side of (18). We then check the condition (17) again and, if it does not hold, we find the highest trap count in the remaining sum ${ }^{N_{2}}\left(C_{i}-S_{t h}\right)$ and repeat our analysis of (18) as above till the condition $i=1$

(17) becomes true. It is obvious from (17) that the number $T$ of traps marked for pesticide application can only be $T \leq N_{2}$ and we minimise the number $T$ by selecting a trap with the highest trap count at every step of our algorithm.

The practical application of the above approach requires that the traps are ordered by the size of the trap count in the descending order and the average trap count $S$ is calculated according to (1). If the average trap count is above the threshold $S_{t h}$, the trap with the highest trap count is marked to be targeted. The trap count in that trap is then considered as 
zero and the average trap count $S$ is recalculated. If the new average trap count in (1) is still 


\begin{tabular}{|c|c|c|}
\multicolumn{3}{c}{$a$} \\
\hline $\mathbf{1 1}$ & $\mathbf{2 0}$ & $\mathbf{2}$ \\
\hline 31 & $\mathbf{1}$ & 0 \\
\hline 8 & 4 & 6 \\
\hline
\end{tabular}

$\mathrm{b}$

\begin{tabular}{|c|c|c|c|c|c|c|c|c|c|}
\hline$i$ & 1 & 2 & 3 & 4 & 5 & 6 & 7 & 8 & 9 \\
\hline$C_{i}$ & $\mathbf{3 1}$ & $\mathbf{2 0}$ & $\mathbf{1 1}$ & 8 & 6 & 4 & 2 & $\mathbf{1}$ & 0 \\
\hline
\end{tabular}

Table 10: (a) Example trap counts in a $3 \times 3$ sampling grid. (b) New number $i$ is assigned to each trap as the trap counts $C_{i}$ are ordered in the descending order. Traps 1 and 2 with trap count $C_{1}=31$ and $C_{2}=20$ respectively (shown in bold in the table) must have zero trap count to ensure that the average trap count is $S<S_{t h}=5$. Hence those traps are marked for pesticide application (see details in the text).

above the threshold then the trap with highest remaining trap count is marked to be targeted and the above procedure is repeated till we have $S<S_{t h}$.

We illustrate selection of patches with high slug density by considering the model example of trap counts in a hypothetical sampling grid of $3 \times 3$ locations. Let trap counts collected on that grid be as shown in table 10. The total number of slugs in the table is 83 with an average trap count $S=9.22$. Let us set the threshold $S_{t h}=5$. As the average trap count $S$ is above the threshold the first step is to target the highest trap count. The trap counts are reordered in the descending order and traps are renumbered accordingly. The highest trap count of 31 now is in the first trap. Assuming none of the other traps are in the area where pesticide is applied, the trap count of 31 is set to o. Hence the new average trap count is $S=5 \cdot 78$. This is still over the threshold and so the next highest trap count, 20, is targeted giving a new average of 3.56. Since the average trap count is now less than the threshold, we can ignore all remaining traps when applying pesticide. 